\title{
Intertwining personal and reward relevance: evidence from the drift diffusion model
}

\author{
A.Yankouskaya, R. Bührle, E. Lugt, M.Stolte, J. Sui
}

\begin{abstract}
In their seminal paper 'Is our self nothing but reward', Northoff and Hayes (2011) proposed three models of the relationship between self and reward and opened a continuing debate about how these different fields can be linked. To date, none of the proposed models received strong empirical support. The present study tested common and distinct effects of personal relevance and reward values by de-componenting different stages of perceptual decision making using a drift-diffusion approach. We employed a recently developed associative matching paradigm where participants (N =40) formed mental associations between five geometric shapes and five labels referring personal relevance in the personal task, or five shape-label pairings with different reward values in the reward task and then performed a matching task by indicating whether a displayed shape-label pairing was correct or incorrect. We found that common effects of personal relevance and monetary reward were manifested in the facilitation of behavioral performance for high personal relevance and high reward value as socially important signals. The differential effects between personal and monetary relevance reflected non-decisional time in a perceptual decision process, and task-specific prioritization of stimuli. Our findings support the parallel processing model (Northoff \& Hayes, 2011) and suggest that self-specific processing occurs in parallel with high-reward processing. Limitations and further directions are discussed.
\end{abstract}

\section{Introduction}

Studies investigating social perception have agreed that reward and self-relevance are two important social factors driving people's basic behavior. A large body of research demonstrated that both reward and self-relevance enhance perceptual, attentional and executive control processes to achieve more efficient goal-directed behavior (Clithero \& Rangel, 2014; Denny, Kober, Wager, \& Ochsner, 2012; Fossati et al., 2004; Kelley et al., 2002; Rushworth, Noonan, Boorman, Walton, \& 
Behrens, 2011). Recent studies provide compelling evidence for improved behavioural performance in the context of perceptual decision tasks with stimuli linked to personal relevance or monetary gains and that the effects of high reward (versus low reward) mimic those of self $^{1}$ (versus others). For example, similar to the self, high reward enhances matching responses to formerly neutral shapes (Sui, He, \& Humphreys, 2012; Sui, Yankouskaya, \& Humphreys, 2015; Yankouskaya, Sui, Moradi, Rotshtein, \& Humphreys, 2017), is less affected by stimulus degradation (Sui et al., 2012) and facilitates decision processes (Enzi, de Greck, Prosch, Tempelmann, \& Northoff, 2009). Furthermore, an enhanced response to reward and the self has been repeatedly observed in conjunction with elevated neural activations in cortical midline structures such as the ventromedial prefrontal cortex (vmPFC), pregenual anterior cingulate cortex (pgACC) and ventral striatum (D'Argembeau, 2013; Doering et al., 2012; Northoff \& Bermpohl, 2004; Rushworth et al., 2011; Schneider et al., 2008). The similarity in behavioural and neural effects of self and high reward raised the question to what extent these effects reflect each other (Northoff, 2016). The importance of this question stems from a continuing debate about whether the self reflects higher-order features of cognitive processes (Humphreys \& Sui, 2016; Sui, 2016), basic functions of the brain’s spontaneous activity (Bai et al., 2016; Conway, Pothos, \& Turk, 2016; Lane, Duncan, Cheng, \& Northoff, 2016; Northoff, 2016), or the binding levels of processing (Sui \& Humphreys, 2015a). Comparing the effects of self-relevance with the effects triggered by other basic motivational functions such as reward, group membership or emotions provides a way to 'trace the trajectory of self'.

Northoff and Hayes (2011) proposed three models of the relationship between the self and reward: (i) the integration model assuming overlap between self and reward, (ii) the segregation model where value assignment and self-specificity assignment are regarded as different processes that are regionally and temporally segregated, and (iii) the parallel processing model posits that different aspects of self-specific processing may occur in parallel with aspects of reward- related

\footnotetext{
${ }^{1}$ Following Christoff, Cosmelli, Legrand and Thompson (2011) we define the term 'self-relevance' as a processing requiring one to evaluate or judge some feature in relation to self. The self in this context reflects our ability to form a mental association between self and an object and includes a memorial component (Gallagher, 2000).
} 
processing at some levels, but assumes a complex relationship between self and reward with multiple interactions across the continuum. Although recent work has made progress in evaluating behavioural effects of self and reward (Madan, Ludvig, \& Spetch, 2014; Madan \& Spetch, 2012; Sui \& Humphreys, 2015c; Sui, Ohrling, \& Humphreys, 2016; Sui et al., 2015; Zhan et al., 2016), none of these models received convincing empirical support (for a review see Northoff, 2016). This is, particularly, due to methodological limitations such as different experimental tasks and procedures being used to examine the effects of personal relevance and reward on cognitive processes. For example, during reward task subjects might perform a complex gambling task, where they could either win or lose whereas, during personal relevance blocks, subjects indicated whether a stimulus was of high or low relevance to them (e.g., Enzi et al., 2009).

Humphreys and Sui (2012) introduced a robust experimental paradigm to test behavioural effects of self and reward using an associative matching procedure, which allows performance to be directly compared across the tasks. The associative matching procedure requires participants, first, to form mental associations between geometric shapes and labels referring personal relevance (e.g., circle - you, hexagon - friend, rectangle - stranger) in personal task, and between geometric shapes and labels referring different reward values (e.g., square - $£ 9$, diamond - $£ 4$, triangle - $£ 1$ ) in reward task. Immediately after, they perform a matching procedure by indicating whether the displayed shape-label pairings are correct or incorrect. A unique aspect of this design is that it triggers common cognitive processes underlying a mental synthesis of a neutral object and a person or reward value, enabling direct comparisons between the effects of personal relevance and reward on perceptual decision (Sui et al., 2012).

Using the associative matching paradigm, previous studies reported two main findings of the effects of self-relevance and reward. First, responses to associations with high salient stimuli (e.g., self, high reward) were faster and more accurate compared with objects associated with others or low reward (Sui \& Humphreys, 2015b). Biased responding to self- and high reward-associations occurred even when stimuli were visually degraded (Sui et al., 2012). Second, no correlation was observed between biased responding to self- (defined as [RTother - RTself]) and high reward(defined as [RTlow reward - RThigh reward]) association indicating that self- and high reward-biases 
may reflect different underlying origins (Sui \& Humphreys, 2015c; Sui et al., 2015). The last finding received additional support from a study where participants had to link self and friend (high reward and low reward) with two different shapes and then classified a single or redundant (two) stimuli representing the self or the friend (high reward or low reward) (Yankouskaya et al., 2017). In line with previous research, there were comparable patterns of self- and high reward-biases observed, but the analysis of capacity coefficient (Townsend \& Eidels, 2011; Townsend \& Wenger, 2004) further revealed that self-associations, but not high-reward associations generated mean redundancy gains with identical shapes and only self-associations consistently violated the independent processing model and produced evidence of super-capacity processing. In contrast, there was an apparent qualitative shift in the processing of reward-related items, where limited capacity processing was evident. These results point out the different architecture of processing shapes 'tagged' with selfrelevance and reward values (Yankouskaya et al., 2017).

It has to be noted that when personal relevance and reward are linked to each other experimentally, the interplay between self- and reward-associations may occur. For example, when shape-label pairs (e.g., circle-you, square-friend, triangle-stranger) were linked to different reward values (e.g., £8, £2, £0) (Sui \& Humphreys, 2015), responses to self-related pairs were less affected by reward associations, but high reward facilitated other personal pairs (friend and stranger) relative to the same personal pairs linked to low reward. The interplay between between self and reward processing was reported in a recent EEG-study were participants performed a head orientation judgment task (left/right) with face images (i.e., self, friend or stranger) presented after a cue stimulus (e.g., monetary reward or blank paper) (Zhan et al., 2016). The data showed facilitation effects of reward on self-faces at later controlled attention stages (indexed by the P3 and LPP components), but not at an early automatic attention stage (e.g., indexed by the N2 component).

Although the facilitation effects indicate possible relationship between personal relevance and reward value, the key question about common and distinct effects of personal relevance and reward values on perceptual decision making is still unanswered. Answering this question will inform current models of the relationship between self and reward and may have important implications for clinical studies. For example, in understanding impaired self and reward processing 
in Autism Spectrum Disorders (ASD) (e.g., Chakraborty \& Chakrabarti, 2015; Scott-Van Zeeland, Dapretto, Ghahremani, Poldrack \& Bookheimer, 2010), social anxiety (Maresh, Allen \& Coan, 2014) and in patients with anterior and medial cortical lesions (Farinelli, Panksepp, Gestieri, Leo, Agati, ... Northoff, 2013). Here we directly address the question by mapping cognitive processes underlying responses to personal - and reward-related stimuli using signal detection and diffusion model approaches.

The diffusion model approach (Ratcliff, Smith, Brown, \& McKoon, 2016; Voss, Nagler, \& Lerche, 2013) assumes that perceptual decision making is based on a stochastic accumulation of incoming sensory information. Specifically, the difference in sensory evidence supporting two response alternatives (e.g., 'correct pairing' vs. 'incorrect pairing') accumulates over time to present an internal decision boundary, which determines the choice. This accumulation stops once enough evidence has been sampled, and a decision is made. By estimating a set of parameters of the diffusion process (such as pre-decision encoding, the rate of information accumulation, the execution of the selected response), this approach enables comparisons between ongoing cognitive processes for different conditions (Voss, Rothermund, \& Voss, 2004). Previously, drift diffusion modelling was applied to data from a number of studies on perceptual decision making in reward processing (Goldfarb, Leonard, Simen, Caicedo-Nunez, \& Holmes, 2014; Moustafa et al., 2015). There was no study, however, using the diffusion model approach to investigate the differences between the effects of self- and reward-associations on perceptual decision processes.

\section{Overview and hypotheses}

The core interest of the present study is to investigate the commonalities and differences between the effects of self- and reward-associated stimuli on perceptual decision making. The null hypothesis in our study is that there will no reliable differences between the effects of personal relevance and reward values on underlying perceptual decision making. Accepting the null hypothesis will support the integration model (Northoff \& Hayes, 2011). It has to be noted that the effects of personal relevance and reward values can be considered at two levels: (i) the task level where we manipulate the relatedness of a stimulus to persons or reward incentives; (ii) the condition level where we assign a stimulus to a specific person or a concrete reward value. Strong support for 
the integration model will be obtained if we observe common effects at both levels. If personalrelevance and reward values have differential effects on parameters of the decision process, the results will provide evidence for the segregation model. Alternatively, some parameters of decision making process may show common patterns for personal and reward tasks, but have stimulusspecific effects within each task. Particularly, based on the results of previous studies (Sui \& Humphreys, 2015b) we expect no effect of task (personal, reward) on accuracy and RT performance and no significant differences between starting point of the evidence accumulation process in each task. However, it is plausible to assume that personal and reward associations may differentially affect drift rate because they have a different exertive draw on attention. For example, it was demonstrated that 'self-cued' stimuli automatically capture attention (Yankouskaya, Palmer, Stolte, Sui, \& Humphreys, 2016), but there is no evidence for automatic reward processing.

To test the hypotheses, we carried out an experiment where participants performed two separate associative matching tasks - one using stimuli associated with persons and the other one with stimuli associated with reward values. Previous studies employing associative matching procedure used two- (Sui et al., 2012; Sui et al., 2016) or three-item (Sui \& Humphreys, 2015a) associations in each task (e.g., circle - you, square-friend, pentagon-stranger in the personal task; rectangle-£8, triangle-£4, diamond-£1 in reward task). It was demonstrated that participants could reliably distinguish between the self and others (e.g., friend and stranger) when responding to personal shape-label pairings and between high (e.g., £8) and low reward values (£4, £1) when responding to reward shape-label pairings. Although the difference between others (i.e., friend and stranger) or low reward values (i.e., $£ 4$ and $£ 1$ ) yielded weaker effects, the data from a number of studies consistently showed a significant linear trend across stimuli in both tasks. The trend indicates that either self and high reward may serve as an anchor point in decision making (e.g., Sui \& Humphreys, 2013). If this holds, it would be interesting to test whether people use similar decision criteria in personal and reward tasks and whether accumulation of information for personal associations is similar to reward-associations. 
To test these assumptions, we used 5-item associations in each task (self, mother, partner, friend, stranger in personal association task, and 9x, 7x, 5x, 3x, 1x in reward association task) which enables us to examine the decision-making process in each task.

\section{Method}

Subjects

Forty young adults ( $M=20.3, S D=2.56)$ (25 females) participated in the present study. All participants were self-identified as being in a romantic relationship. They were recruited within colleges and departments at the University of Oxford. The subjects reported no neurological conditions and had normal or corrected-to-normal vision. This experiment was approved by Central University of Oxford Research Ethics Committee (CUREC). All participants provided informed consent.

Apparatus, stimuli and procedure

Participants performed an associative matching procedure where stimuli associated with personal and reward relevance were assigned to separate blocks presented in counterbalanced order. For simplicity, we labelled the blocks as ‘personal task' and 'reward task'. Ten geometric shapes were randomly assigned to labels representing five persons (me, partner, mother, friend, stranger) and five reward values $(\mathrm{x} 9, \mathrm{x} 7, \mathrm{x} 5, \mathrm{x} 3, \mathrm{x} 1)$. An instruction for each task was displayed on the screen asking participants to remember five shape-label pairings (e.g., me-circle, partner-square, mother vertical rectangle, friend-pentagon, stranger-horizontal oval in the personal task; x9-triangle, x7vertical oval, x5-diamond, x3-upside-down triangle, x1- horizontal rectangle in the reward task). An experimental design and example of stimuli are displayed in Figure 1. In the reward task, ' $x$ ' corresponded to a certain monetary amount on each trial (£0.01), for example, 'x9’ was rewarded 9 times for correct responses as much as ' $\mathrm{x}$ '. Participants were aware that they would receive the total sum of the corresponding bonuses for correct responding in the reward task. They were not limited in the amount of time to memorize the associations, but in average, they spent 2-3 minutes in each task. 


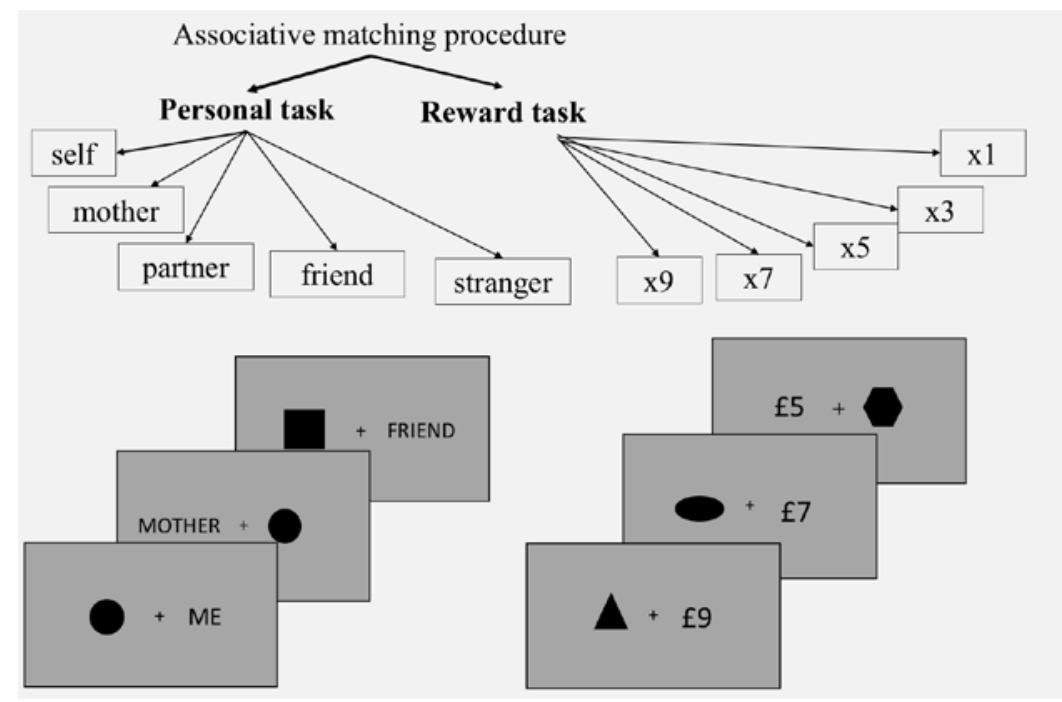

Fig. 1 An experimental design and example of stimuli in personal and reward tasks.

The instruction stage was followed by a short training ${ }^{2}$ (20 practice trials) and the subsequent experimental stage where participants had to make an accurate and fast judgment whether a displayed shape-label pairing was correct (half of the trials) or incorrect. The pair either conformed to the written instructions given at the beginning of the experiment or it was a recombination of a label with a different (non-matching) shape. The presentation of shape-label pairs was randomised across trials. Subjects responded to a shape-label stimulus by pressing the corresponding key (i.e., 'n' and 'm' keys balanced across participants for responses 'correct pairing' and 'incorrect pairing'). Feedback on accuracy (words ‘Correct!' or 'Incorrect!') and overall response time was provided after each trial in the training. In the experimental stage, participants performed eight blocks (four blocks for personal task and four blocks for reward task) of 150 trials yielding in total 600 trials in each task (60 trials per condition; five matching and five nonmatching conditions). No feedback was provided during the experimental stage.

On each trial a central fixation cross (covering $0.8^{\circ} \times 0.8^{\circ}$ visual angle) appeared for $500 \mathrm{~ms}$ followed by a shape (covering $3.5^{\circ} \times 3.5^{\circ}$ of visual angle)-label (covering $1.76^{\circ} 2.52^{\circ} \times 1.76^{\circ}$ of visual angle) stimulus for $100 \mathrm{~ms}$ and a blank interval (1000 ms) for participants’ response (Figure 1). If a subject made no response during the blank interval, a feedback 'too slow' was appeared on the screen and the stimulus would be presented again by the end of the block.

2 The number of trials in the training stage was determined by previous studies (Sui et al., 2012) and a pilot study with 5 participants. 
The experiment was run on a PC with a 22" monitor (1920 x 1080 pixels) at 60Hz, using Eprime software (Version 2.0) to present the stimuli and record responses.

At the end of the experimental stage, we had participants perform a modified version of a paper-and-pencil projective technique (Sui \& Humphreys, 2015c). We asked participants to indicate the personal distance between two people by making two marks on a straight line (i.e., self and mother, self and friend, self and partner, self and stranger). The instruction was: "Please, mark two points on the following lines to indicate whether the two fall in a relation to each other. The first mark refers to the first person, and the second mark represents the second person”. There were 10 trials per pairing (50 trials in total) presented in random order. The physical distance between the marks was averaged across each pairing. The relative personal distance between self and stranger scaled by the distance between self and mother (self and partner, self and friend) was calculated for each participant and served here as individual indices of the personal distance between the participant and others.

Analyses

All data files are deposited on the Open Research data repository (insert the link here).

Prior entering data into analyses, we removed outliers defined as RTs shorter than $150 \mathrm{~ms}$ (Ratcliff, 2015) which accounted for less than $0.4 \%$ of responses for each participant. Data from one participant were removed due to computer failure in the reward task, and the remaining 39 individual data sets were entered into four analyses.

Accuracy and RT performance. Matched and mismatched pairs were analysed separately due to different responses being made in these cases. We calculated the proportion of correct responses for personal and reward associations and report the overall accuracy performance.

Three analyses were performed on RT data. To obtain a general idea about the difference in RT performance between the task we carried out a paired sample $t$-test. The second analysis examined the effects of stimulus (personal or reward associations) in each task and addressed the question of whether responses to personal and reward associations generate a linear trend across the stimuli using one-way repeated measured ANOVAs and polynomial contrasts. In the third analysis, reaction time data were modelled using a mixed-effects approach. The advantage of using the 
mixed-effect modelling here is that it accounts for inherent dependencies between stimuli due to repeated measures and individual variations in generating associations with personal and reward relevance. For example, stimulus-specific idiosyncrasies may be observed within subject's data due to individual experience in relationship with close, familiar and unfamiliar people. Similarly, subject's socio-economic status may affect individual's value of the amount of the reward.

The mixed-effect approach is based on partitioning error terms in a classical regression model into the usual residual error term plus a number of random effect terms accounting for dependencies in the data by adjusting the predicted values of the model separately for each level of the grouping factor (Task). We used the lme4 and nlme packages to perform the mixed-effect model analysis in R-programming (R Development Core Team, 2013; Baayen, Davidson \& Bates, 2008).

In our experimental design, factor Stimulus is nested within Task factor. A fitted model included response time as a depended variable, fixed effects of Task and two random effect terms: (i) random Stimulus intercepts and (II) random intercepts and Task slopes across Subject. It has to be noted that both fixed effects and random variance components are of substantive theoretical interest here. Particularly, by taking into account the random effects of both Subject and Stimulus and allowing the effects to vary randomly with respect to Subject, we may expect no effects of Task. However, if the effects do exist, analysis of the random variance components may help to explore whether and how associations with personal relevance and reward values contribute these effects.

The contribution of random effects was estimated using the Likelihood Ratio Test (Judd, Westfall, \& Kenny, 2012). The likelihood ratio statistic is equal to two times the difference of the log-likelihoods of two models where one model includes a parameter of interest (fitted model), and the second model (null-model) does not contain the parameter of interest. Normality in the distribution of the residuals of final models was assessed using quantile-quantile plots.

$R T$-gains. We examined the magnitude of the facilitation effects in personal task and addressed the question of whether the advantage in responding to shapes associated with self, close and familiar people generates a linear trend. Similar, we assessed RT advantage for high reward values compared to low reward values. 
Sensitivity index. Using a signal detection approach (Stanislaw \& Todorov, 1999; Witt, Taylor, Sugovic, \& Wixted, 2015), we then computed the sensitivity index (d') for each association within the tasks. The sensitivity index which characterized the sensory process was calculated from the HR (Hits for matched pairs) and FAR (False Alarms for mismatched pairs containing the same shape) as following: $\mathrm{d}^{\prime}=\mathrm{zHR}-\mathrm{zFAR}$, where zHR and zFAR are the z-score transforms, based on the normal distribution, of the HR and FAR probabilities. Assessing the sensitivity index as a relatively stable property of the sensory process provides us with an initial idea of whether RT differences between conditions reflect differences in perceptual sensitivity to stimuli (Witt, Taylor, Sugovic, \& Wixted, 2015).

Drift diffusion modelling. To get a more thorough understanding of cognitive processes underlying the effects of personal- and reward-associations on behavioural performance, we used the drift diffusion modelling approach (Ratcliff \& McKoon, 2008) that enables statistical separation of different components of a speeded binary decision process. The drift diffusion model assumes that decisions are based on processing of information accumulated over time. This process moves between two boundaries, which are linked to the two possible decisional outcomes (e.g., CR'correct response’ and IR - 'incorrect response’). Therefore, evidence supporting CR outcome drives the process towards boundary CR (e.g., upwards), while evidence supporting the IR decision drives the process towards boundary IR (e.g., downwards). As soon as one of these thresholds is reached, the corresponding response (e.g., pressing 'n' vs. 'm' key) will be triggered and executed. The components of the decision process are represented by different parameters of the model. A short description of the parameters is presented in Table 1 (Voss, Voss, \& Lerche, 2015). Here we aim to examine the effects of stimuli manipulation in the personal and the reward tasks on different components of the decision process by comparing the parameters between tasks and conditions.

Table 1 Parameters in the drift diffusion model

Parameter Description

\footnotetext{
a The width of the interval between decision thresholds; quantifies the amount of evidence that must accumulate before a response is initiated

$v \quad$ The strength (and direction) of the systematic influence on the diffusion process.

$z \quad$ The starting value of the process. If the starting point is closer to the upper threshold
} 
(i.e., $\mathrm{z}>\mathrm{a} / 2$ ), the mean RTs of the response connected to the upper threshold will be smaller than the mean RTs of the lower threshold.

to The duration of all extra-decisional parts of the response time. This parameter sums up all extra-decision processes, that is pre-decision components (e.g., stimulus encoding) and post-decision processes (e.g., response execution). Importantly, the t0 parameter is conceptually independent of all processes of response selection (which are captured by the diffusion process)

$s z$, sv, st0 Inter-trial variability for the starting value (z), drift diffusion and non-decision time respectively.

The diffusion-model data analysis was conducted with fast-dm (Voss et al., 2015). To test the effects of experimental manipulations in personal task and reward tasks on parameters of the diffusion process, we allowed two parameters ( $v$ and $t 0)$ to vary between conditions in each task. By allowing the drift rates $(v)$ to vary between conditions we expect to gain an idea of how fast information enters the decision process for shape-label associations in each task. On the other hand, it is plausible to assume that depending of individual importance of personal and reward associations, the strength of representations which are stored in memory may vary across conditions within a participant. Thus, the response execution may be facilitated or delayed due to response preparation or interference which would map on non-decisional time (t0). For a more robust estimation of the parameters, we set inter-trial-variability of drift (sv) and starting point variability (szr) to zero. The starting point (z) and threshold separation (a) were fixed to the same values across conditions within a participant. We report z/a instead of $\mathrm{z}$ because it can be interpreted as a measure of decision bias (Voss et al., 2015). Values of z/a greater than 0.5 indicate a bias toward the response associated with the upper boundary, whereas values less than 0.5 indicate a bias toward the response associated with the lower boundary.

Our primary interest focused on matched ${ }^{3}$ trials in each task. For the present analyses, the diffusion model was fitted to individual reaction time distributions for five matched trials in personal (self, mother, partner, friend, stranger) and reward (9x, 7x, 5x, 3x, 1x) tasks. The upper (lower) threshold was assigned to correct (incorrect) responses. In total, we estimated parameters for 39

\footnotetext{
${ }^{3}$ Fitting the diffusion model to responses in mismatched trials is less meaningful, because different labels (e.g., mother, partner, friend, stranger) paired with a shape (e.g., for self) may contaminate accumulating evidence toward decision boundaries
} 
models in each task. Each model was based on 300 responses (60 trials x 5 conditions) minus excluded responses, if any.

We estimated the model fit by using the Kolmogorov-Smirnov (KS) test. The estimation procedure enables us to assess the degree of correspondence between theoretical and empirical distributions based on T-statistics (i.e., the maximum vertical distance between the empirical RT distribution and the predicted time distribution) (Voss et al., 2013). A significant result (i.e., one below $\mathrm{a}=.05$ ) indicates that observed data largely deviates from prediction. The model fit (KS-test) was computed for each participant and each condition. To estimate the overall fit of the models to the data, the number of significant KS tests $(<.05)$ were entered into a binomial test with parameters of n (number of tests) and p () (Voss \& Voss, 2007).

Personal distance and RT-gains. Finally, we examined the relationship between the index of the personal distance and RT differences between conditions in the personal task. Particularly, we were interested in whether subjective experience of personal distance to a mother, partner and friend can predict the difference in RT performance between self and mother, partner and friend. Three separate linear regression analyses were conducted with the index of the personal distance as the predictor and RT difference as the dependent variable.

Additional analysis. To quantify evidence in favour of the null-hypothesis (H0), we calculated a Bayes factor (BF) for all crucial contrasts using the JZS-approach (Rouder, Speckman, Sun, Morey, \& Iverson, 2009; Rouder, Speckman, Morey, \& Province, 2012). The JZS Bayes Factor which provides the odds ratio for the null/alternative hypotheses $\left(B F_{01}\right)$ given the data is based on the combination of the Cauchy on effect size and the Jeffreys prior on variance (Rouder et al., 2009). The JZS Bayes factor was computed with version 0.9.8 of the BayesFactor package for $\mathrm{R}$ using ttest.tstat function ${ }^{4}$ (Morey, Rouder, Pratte, \& Speckman, 2011). Here we report the raw $\left(B F_{01}\right)$ to communicate evidence for the H0. When appropriate, the inverse BF was calculated to provide evidence for the alternative (H1) hypothesis and reported as (inverse BF).

\footnotetext{
${ }^{4}$ As the ttest.tstat function returns the $\log (\mathrm{e})$ Bayes factor against the null hypothesis, we applied the conversion formula (1/exp(BF)) to obtain the raw values for BF.
} 


\section{Results}

Accuracy performance

Proportions of correct and incorrect responses in personal and reward tasks are displayed in Figure 2. Participants were accurate in both tasks $(M=0.86, S D=0.03 ; M=0.87, S D=0.02$, in personal and reward task respectively). There were no significant differences in accuracy between personal and reward tasks for matched $\left(t(38)=0.63, p=.53, d z=0, B F_{01}=6.61\right)$ and mismatched pairs $\left(t(38)=0.45, p=.65, d z=0, B F_{01}=7.26\right)$.

Previous studies reported a linear trend in accuracy performance in personal task using three-item associations (Sui, Ohrling, \& Humphreys, 2016). Here we tested whether a linear trend is also held for five-item associations. Polynomial contrasts showed that in both tasks the proportion of correct responses for matched pairs decreased linearly with decreasing personal relevance $(F(1,38)$ $=36.28, \mathrm{MSE}=0.352, p<0.001)$ and reward value $(\mathrm{F}(1,38)=20.35, \mathrm{MSE}=0.17, p<0.001)$

(Figure 2). The polynomial contrasts for mismatched trials in both tasks did not show a linear trend $(\mathrm{F}(1,38)=1.87, p=.19 ; \mathrm{F}(1,38)=0.93, p=.79)$.

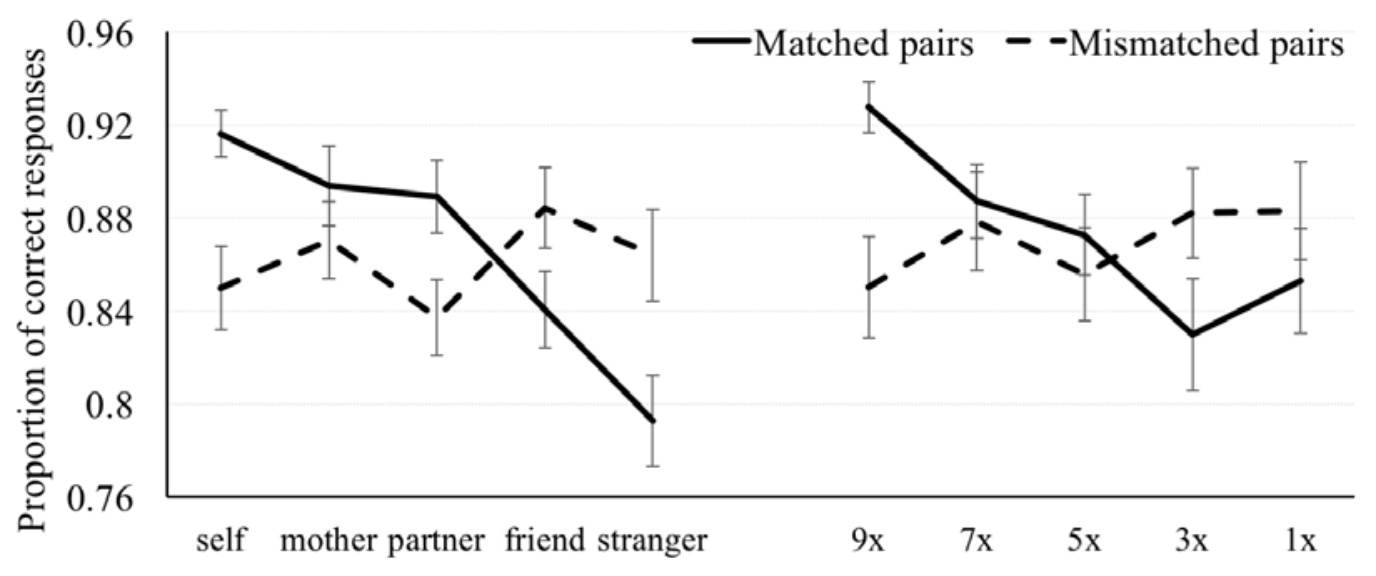

Fig. 2 A proportion of correct responses for matched and mismatched pairs in personal and reward tasks. Error bars represents +/- SEM

\section{RT performance}

To gain a general idea about RT performance, we first examined the difference in RT between personal and reward tasks using paired sample $t$-tests. The results showed that for both 
matched and mismatched pairs responses in the personal task were slower compared to the reward task $((t(38)=7.89, p<.001, d z=1.26 ; t(38)=8.54, p<.001, d z=1.36)$ (Figure 3, A).

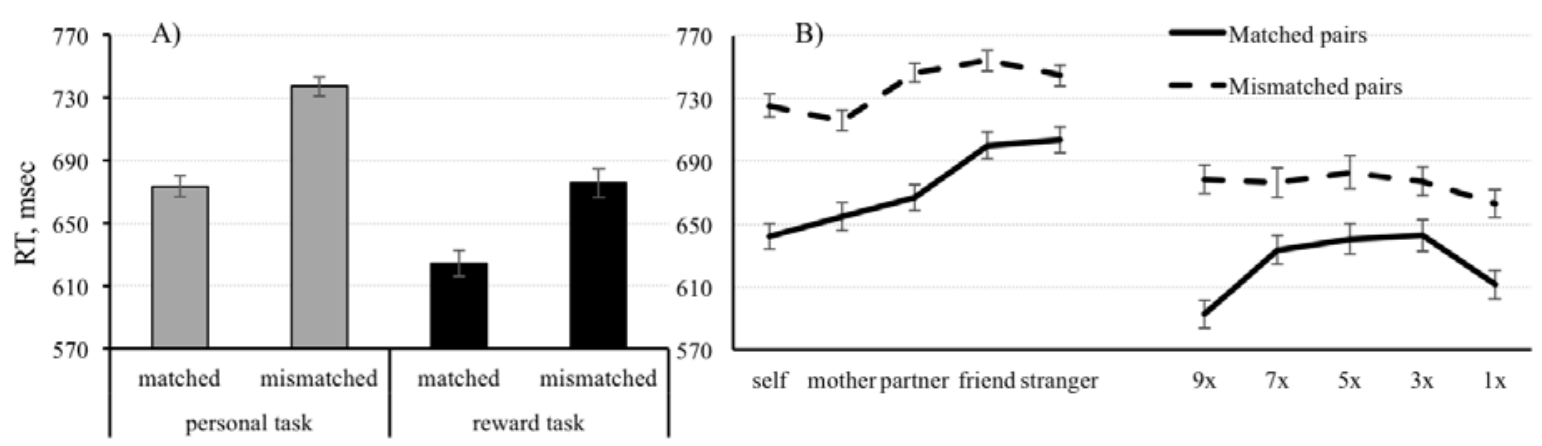

Fig. 3 Mean reaction times (RT) for matched and mismatched pairs in personal (A) and reward (B) tasks. Error bars represents +/- SEM

We then examined the effects of Stimulus in each task and assessed whether responses to personal and reward associations generate a linear trend across the stimuli using one-way repeated measured ANOVAs. In total, four separate ANOVAs were performed (for matched and mismatched pairs in personal and reward tasks).

Personal task. There was a main effect ${ }^{5}$ of Stimulus (self, mother, partner, friend, stranger) for matched pairs $(\mathrm{F}(4,152)=23.18, \mathrm{MS}=29190,79, p<.001)$. A polynomial contrast showed a strong linear trend in increasing RT across the continuum self-mother-partner-friend-stranger (polynomial contrast $\mathrm{F}(1,38)=72.43, \mathrm{MSE}=110,616, p<0.0001)$. Post Hoc analysis with Bonferroni corrections for multiple comparisons indicated no significant differences between RT for self, mother and partner $\left(t(38)=1.7, \mathrm{p}=.9, d z=0.27, B F_{01}=2.05 ; t(38)=2.7, p=.085, d z=0.43\right.$, $\left.B F_{01}=0.31 ; t(38)=1.5, p=1.0, d z=0.24, B F_{01}=2.75\right)$. It has to be noted that an estimated Bayes factor (null/alternative) for the self-partner comparison indicates that the difference between these conditions was 3.23 times more likely to occur under the alternative hypothesis.

Responses to shapes associated with friend and stranger for matched pairs were slower compared to shapes associated with self, mother and partner $(t(38)=7.6, p<.001, d z=1.21 ; t(38)=$ $6.5, p<.001, d z=1.04 ; t(38)=7.2, p<.001, d z=1.15 ; t(38)=5.9, p<.001, d z=0.94 ; t(38)=4.3$,

\footnotetext{
${ }^{5}$ Sphericity is assumed unless otherwise specified.
} 
$p=.001, d z=0.69 ; t(38)=4.6, p<.001, d z=0.74)$. Thus, these results provide further support for a linear trend across the stimuli in personal task.

For mismatched pairs, there was a main effect of Stimulus $(\mathrm{F}(3.05,115.9)=26.44, \mathrm{MSE}=$ 9913,79, $p<0.001$ (degrees of freedom were corrected using Greenhouse-Geisser estimates of sphericity)). A polynomial contrast showed a significant linear trend in increasing response time in the continuum self-mother-partner-friend-stranger $(\mathrm{F}(1,38)=37.39$, MSE $=22954,43, p<0.001)$. Post Hoc pairwise comparisons with Bonferroni corrections showed that responses to mismatched pairs containing shapes associated with self and mother were significantly faster compared to the rest of the stimuli $(t(38)=4.2, p=.001, d z=0.67 ; t(38)=5.3, p<.001, d z=0.08 ; t(38)=3.9, p<.004$, $d z=0.62 ; t(38)=3.9, p=.001, d z=0.62 ; t(38)=6.6, p<.001, d z=1.06 ; t(38)=9.2, p<.001, d z=$ 1.47, for partner, friend and stranger respectively) and responses to pairs containing partnerassociated shape were faster compared to stranger $(t(38)=5.9, p<.001, d z=0.94)$. No significant difference was found between friend and stranger $\left(t(38)=0.41, p=.25, d z=0.07, B F_{01}=7.39\right)$ for mismatched pairs. The difference between self and mother $\left(t(38)=2.3, p=.26, d z=0.36, B F_{01}=\right.$ 0.71) also did not reach a significance level. A Bayes factor provides insufficient evidence for either the null and the alternative hypotheses.

Reward task. A repeated measures ANOVA showed a main effect of Stimulus (x9, x7, x5, $\mathrm{x} 3$, $\mathrm{x} 1)$ for matched pairs $(\mathrm{F}(1,38)=18.77, \mathrm{MSE}=17852,31, p<0.001)$ and a strong quadratic trend (a polynomial contrast, $\mathrm{F}(1,38)=70.11, \mathrm{MSE}=61088,64, p<0.0001)$. Post Hoc pairwise comparisons with Bonferroni corrections revealed no significant differences between x7, x5 and x3 reward values $\left(t(38)=2.4, p=.18, d z=0.38, B F_{01}=0.58 ; t(38)=0.92, p=1.0, d z=0.15, B F_{01}=\right.$ 5.32; $\left.t(38)=1.3, p=1.0, d z=0.21, B F_{01}=3.57\right)$. The difference between the polar ends of the reward continuum (i.e., between $\mathrm{x} 9$ and $\mathrm{x} 1$ ) was non-significant $\left(t(38)=2.2, p=.18, d z=0.35, B F_{01}\right.$ $=0.86$ ). The Bayes factor was inconclusive for the difference between $\mathrm{x} 7$ and $\mathrm{x} 3$ and $\mathrm{x} 9$ and $\mathrm{x} 1$ reward values.

Responses to either x9 and x1 reward values were significantly faster compared to all intermediate reward values $(\mathrm{x} 7, \mathrm{x} 5, \mathrm{x} 3)(t(38)=5.8, p<.001, d z=0.93 ; t(38)=6.4, p<.001, d z=$ 
$1.02 ; t(38)=6.8, p<.001, d z=1.08 ; t(38)=3.2, p=.001, d z=0.51 ; t(38)=4.2, p<.001, d z=$ $0.67 ; t(38)=4.8, p<.001, d z=0.77)$.

For mismatched trials, there was a significant main effect of Stimulus $(F(4,152)=6.39$, MSE $=2152,85, p<.001)$. A polynomial contrast showed that the data fit either a linear and quadratic trends $(\mathrm{F}(1,38)=9.88, \mathrm{MSE}=3568,11, p=.003 ; \mathrm{F}(1,38)=9.79, \mathrm{MSE}=3595,74, p=$ .003, respectively). Post Hoc pairwise comparisons revealed that responses to a shape associated with the lowest reward value (i.e., x1) were significantly slower compared to the rest of stimuli $(t(38)=3.8, p=.004, d z=0.61 ; t(38)=3.2, p=.02, d z=0.51 ; t(38)=3.9, p=.005, d z=0.62$ $t(38)=3.2, p=.03, d z=0.51)$. No significant differences were found in pairwise comparisons between $\mathrm{x} 9, \mathrm{x} 7, \mathrm{x} 5$ and $\mathrm{x} 3$ rewards $\left(t(38)=0.82, p=1.0, d z=0.13, B F_{01}=5.78 ; t(38)=.98, p=1.0\right.$, $d z=0.16, B F_{01}=5.04 ; t(38)=0.35, p=.86, d z=0.06, B F_{01}=7.55 ; t(38)=1.5, p=.56, d z=0.24$, $B F_{01}=2.75 ; t(38)=0.20, p=.78, d z=0.12, B F_{01}=7.86 ; t(38)=1.2, p=.31, d z=0.19, B F_{01}=$ 4.01).

The mixed-effect modeling. Visual inspection of residual Q-Q plots did not reveal any obvious deviations from homoscedasticity or normality (insert link to Supplementary Material). Fitting a model with fixed (Task) and random effects (Stimulus intercepts and by-Subject adjustment to Task intercepts and slopes) for matched pairs indicates that removing the random effects from the model significantly decreases the goodness of fit as indicated by likelihood ratio tests (Table 2).

Table 2 Mixed-effects modelling of correct responses in matched pairs ( $\chi 2$ - Chi-Square value, SD variability expressed as a standard deviation for Variance component, CI - 95\% confidence interval* (min=2.5\%, max=97.5\%), SE - standard error, $t$ - maximum likelihood $t$-test for fixed effects, $d f-$ degrees of freedom using Satterthwaite approximations, $p$-value for the fixed effects and model fit)

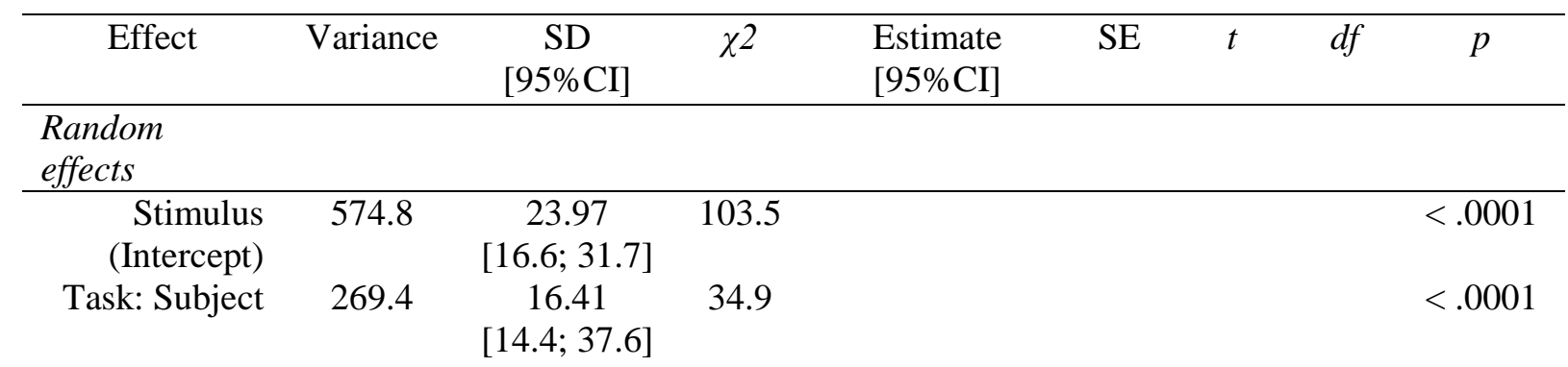


Fixed effects

Intercept

$$
648.77
$$

[628.9; 668.7]

Task

$$
24.64
$$

$[8.4 ; 40.8]$

$$
\begin{array}{cccc}
10.2 & 63.29 & 21.5 & <.0001 \\
8.2 & 3.6 & 9.8 & <.01
\end{array}
$$

*95\% CI was computed based on approximate asymptotic estimates using the likelihood profile method with R package lme4

Effect (contrast) coding was used to code the fixed factor (.5 personal task; -.5 reward task) and the difference between the levels of Task was computed as the product of these codes. This analysis revealed a significant effect of Task such that responses were faster for reward associations compared to personal associations (Table 2).

To explore the random effect of Stimulus (nested within Task) we computed and plotted the conditional mode for each stimulus (i.e., the difference between the average predicted response for fixed-effect values and the response predicted for a particular stimulus). The results presented as a dotplot of prediction intervals for the random effects of Stimulus for matched pairs (Fig 4, a) indicate differential contribution of personal and reward associations to the random effect. Specifically, all personal associations excluding associations with partner showed random effects distinguishable from zero. In contrast, prediction intervals on the random effects of x1, $x 7$ and marginally x5 reward associations overlap zero (Figure 4a).

a)

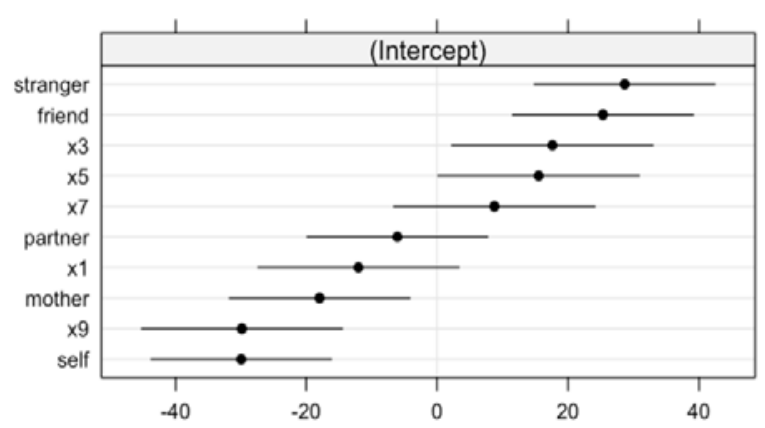

b)

STIMULUS

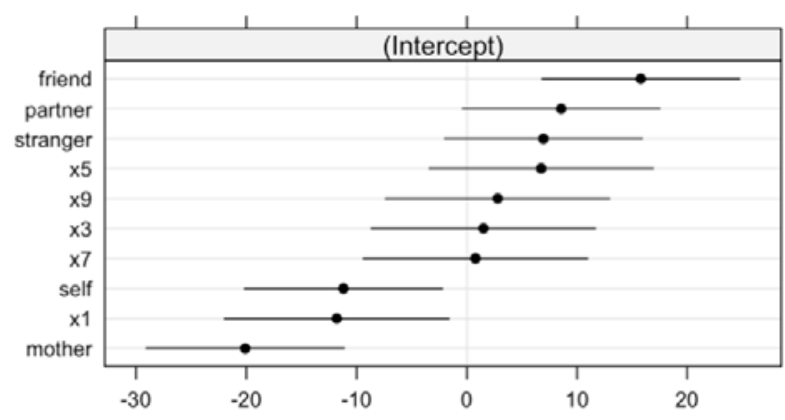

Fig. 4 Estimate (black dots) and 95\% prediction intervals (horizontal lines) on the random effects of Stimulus for (a) matched pairs and (b) mismatched pairs in personal and reward task. 
Fitting a model with fixed (Task) and random effects (Stimulus intercepts and by-Subject adjustment to Task intercepts and slopes) for mismatched pairs showed that, similar to matched pairs, removing the random effects from the model significantly decreases the model fit (Table 3).

Table 3 Mixed-effects model results for correct responses in mismatched pairs ( $\chi 2$ - Chi-Square value, SD - variability expressed as a standard deviation for Variance component, CI - 95\% confidence interval* $(\min =2.5 \%$, max $=97.5 \%), \mathrm{SE}$ - standard error, $t$ - maximum likelihood $t$-test for fixed effects, $d f$ - degrees of freedom using Satterthwaite approximations, $p$-value for the fixed effects)

\begin{tabular}{|c|c|c|c|c|c|c|c|c|}
\hline Effect & Variance & $\begin{array}{c}\text { SD } \\
{[95 \% \mathrm{CI}]}\end{array}$ & $\chi^{2}$ & $\begin{array}{l}\text { Estimate } \\
{[95 \% \mathrm{CI}]}\end{array}$ & SE & $t$ & $d f$ & $p$ \\
\hline \multicolumn{9}{|l|}{$\begin{array}{l}\text { Random } \\
\text { effects }\end{array}$} \\
\hline $\begin{array}{r}\text { Stimulus } \\
\text { (Intercept) }\end{array}$ & 145.6 & $\begin{array}{c}12.07 \\
{[7.3 ; 19.8]}\end{array}$ & 83.2 & & & & & $<.0001$ \\
\hline Task: Subject & 470.4 & $\begin{array}{c}21.69 \\
{[17.1 ; 27.7]}\end{array}$ & 513.1 & & & & & $<.0001$ \\
\hline Residual & 355.9 & $\begin{array}{c}18.87 \\
{[17.4 ; 20.4]}\end{array}$ & & & & & & \\
\hline \multicolumn{9}{|l|}{ Fixed effects } \\
\hline Intercept & & & & $\begin{array}{c}706.3 \\
{[690.9 ; 721.6]}\end{array}$ & 7.8 & 90.3 & 42.9 & $<.0001$ \\
\hline Task & & & & $\begin{array}{c}30.79 \\
{[20.4 ; 41.1]}\end{array}$ & 5.3 & 5.86 & 22.1 & $<.0001$ \\
\hline
\end{tabular}

In contrast to matched pairs, analysis of prediction intervals for the random effects of Stimulus in mismatched pairs showed that only one reward association (x1) contributes to the random effects of Stimulus. Prediction intervals for three (self, mother and friend) personal associations did not overlap the intercept (Fig 4, b).

RT-gains (facilitation effects)

To examine the facilitation effects of personal relevance and monetary reward values on RT performance, we calculated the magnitude of RT advantage (RT-gain) for self, mother, partner and friend relative to stranger (and for 9x, 7x, 5x, 3x reward values relative to 1x) for each participant. By assessing the relative differences at the individual level, we measured relative sizes of the differential effects of personal relevance and monetary reward values disentangling the individual 
variability in RTs. RT-gains were calculated as following: self (RTstranger - RTself), mother (RTstranger - RTmother), partner (RTstranger - RTpartner), friend (RTstranger - RT friend). Similarly, we defined four RT-gains in reward task - 9x (RT1x - RT9x), 7x (RT1x - RT7x), 5x (RT1x - RT5x), 3x (RT1x - RT3x). RT-gains were computed for matched and mismatched pairs (Figure 5).

One-sample $t$-tests with bias corrected and accelerated (BCa) bootstrapping procedure indicated that in matched pairs, the magnitude of RT-gains for self, mother, partner and x9 reward was significantly greater than zero $\left(t(38)=6.56, p<.001, d z=0.89, \mathrm{MD}^{6}=61.4 \mathrm{msec}\right.$, BCa 95\% CI $[42.1,80.9] ; B F_{01}=6.27 \mathrm{e}-06 ; t(38)=5.93, p<.001, d z=0.95, \mathrm{MD}=48.9 \mathrm{msec}$, BCa 95\% CI [31.5, 65.9], $B F_{01}=4.17 \mathrm{e}-05 ; t(38)=4.62, p<.001, d z=0.74, \mathrm{MD}=36.4 \mathrm{msec}$, BCa 95\% CI [20.9, 52.3], $B F_{01}=0.002 ; t(38)=2.45, p=.02, d z=0.039, \mathrm{MD}=18.7 \mathrm{msec}, \mathrm{BCa} 95 \% \mathrm{CI}[4.49,32.2], B F_{01}=$ 0.52, respectively). Importantly, a Bayes factor provided strong evidence for the alternative hypothesis for self, mother and partner (the inverse ratio $(\mathrm{H} 1 / \mathrm{H} 0)>100)$, however, the evidence for 9x reward value was inconclusive.

A significant RT-gain for self and mother was found even in mismatched trials $(t(38)=3.91$, $p<.001, \mathrm{MD}=19.3 \mathrm{msec}, \mathrm{BCa} 95 \%$ CI $[10.3,28.6], d z=0.63, B F_{01}=0.01 ; t(38)=5.9, p<.001$, $\mathrm{MD}=28.7 \mathrm{msec}$, BCa 95\% CI [18.9, 38.6], $\left.d z=0.95, B F_{01}=4.5 \mathrm{e}-05\right)$. But this was not the case for any reward values (the magnitude of RT-gains were significantly below zero $(t(38)=-3.24, \mathrm{p}<.001$, $\mathrm{MD}=-21.8$ msec, BCa 95\% CI [-34.4, -9.5], $d z=0.52, B F_{01}=0.08 ; t(38)=-4.23, p<.001, \mathrm{MD}=-$ 28.9 msec, BCa 95\% CI [-42.7, -15.6], $d z=0.68, B F_{01}=0.0062 ; t(38)=-4.82, p<.001, \mathrm{MD}=-31.1$ msec, BCa 95\% CI [-43.4, -18.4], $d z=0.77, B F_{01}=0.0011$, for x7, x5 or x3 respectively) (Figure 5).

\footnotetext{
${ }^{6} \mathrm{MD}$ - mean difference
} 


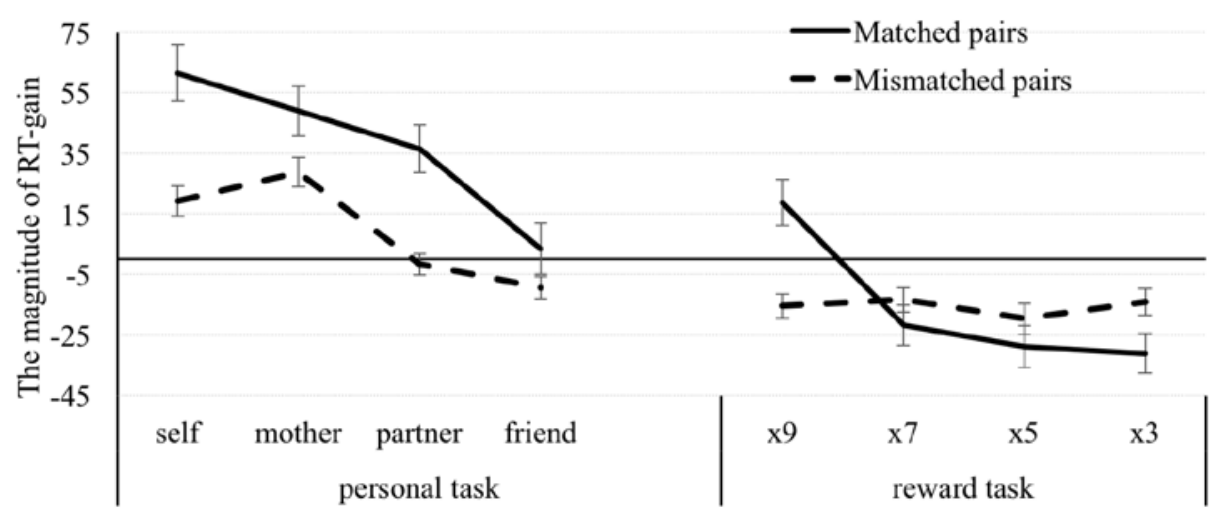

Fig. 5 RT facilitation effects (RT-gains) in the personal and the reward task. The error bars represent SEM +/- 1. The negative values indicate no RT gain. The asterisks showed whether the RT gains were significantly different from 0 (One-sample t-test against 0 ).

To examine the effects of shape category on the magnitude of RT-gain for matched pairs in personal task, a repeated measures ANOVA was carried out. There was a main effect of shape category $(\mathrm{F}(3,114)=32.15, \mathrm{MSE}=24235,52, p<.001)$ and a strong linear trend $(\mathrm{F}(1,38)=56.42$, MSE $=67838,53, p<.001)$. Post Hoc pairwise comparisons with Bonferroni corrections indicated that the magnitude of RT-gains for self and mother was significantly greater than for partner and friend $\left(t(38)=4.3, p=.001, d z=0.69, B F_{01}=0.0051 ; t(38)=5.4, p<.001, d z=0.86, B F_{01}=0.0002\right.$; $t(38)=6.7, p<.001, d z=1.07, B F_{01}=4.12 \mathrm{e}-06 ; t(38)=9.2, p<.001, d z=1.47, B F_{01}=2.9 \mathrm{e}-09$, respectively). No significant differences in RT-gains were found between self and mother $(t(38)=$ $2.31, p=.16, d z=0.31)$, and partner and friend $(t(38)=2.34, p=.15, d z=0.35)$. However, the Bayes factor provides inconclusive evidence for both comparisons $\left(B F_{01}=0.69\right.$ and $B F_{01}=0.65$, respectively).

Finally, we tested whether the magnitude of RT-gain for self can predict the magnitude of RT-gain for high reward. The results showed that the RT-gain for self cannot reliably predict the RT-gain for $\mathrm{x} 9$ reward $\left(B=-0.013, \mathrm{SE}=0.15, t(38)=0.26, p=.79 ; d z=0.041, B F_{01}=7.75\right)$. Perceptual sensitivity

Perceptual sensitivity $\left(\mathrm{d}^{\prime}\right)$ in personal task did not differ significantly from reward task (t(38) $\left.=0.39, p=.69, d z=0.072, B F_{01}=7.44\right)$. 
To examine the effect of Stimulus on $\mathrm{d}^{\prime}$, perceptual sensitivity indices were computed for each condition per participant and submitted to a repeated measures ANOVA for each task separately.

In personal task, there was a main effect of Stimulus (self, mother, partner, friend, stranger) $(\mathrm{F}(4,152)=6.23, p<.001)$ and a strong linear trend in decreasing sensitivity in the continuum selfmother-partner-friend-stranger $(\mathrm{F}(1,38)=17.92, p<.001)$ (Figure 6). Post Hoc pairwise comparisons with Bonferroni corrections showed that sensitivity to self and mother was higher compared to partner $\left(t(38)=2.9, p=.057, d z=0.46, B F_{01}=0.19 ; t(38)=3.4, p=.015, d z=0.54\right.$, $\left.B F_{01}=0.057\right)$ and stranger $\left(t(38)=3.6, p=.01, d z=0.58, B F_{01}=0.034 ; t(38)=3.9, p=.003, d z=\right.$ 0.62, $\left.B F_{01}=0.015\right)$. Although the difference between self and partner did not reach significance level ( $p=.057$ ), the Bayes factor provides evidence in support the H1 hypothesis (the inverse Bayes factor $=5.26)$.

No significant differences was found between shapes associated with self and mother $(t(38)$ $\left.=0.25, p=1.0, d z=0.04, B F_{01}=7.77\right)$, partner and friend $\left(t(38)=0.72, p=1.0, d z=0.12, B F_{01}=\right.$ 6.23), self and friend $\left(t(38)=1.37, p=.18, d z=0.22, B F_{01}=3.27\right)$. The difference between mother and friend $\left(t(38)=1.61, p=.12, d z=0.26, B F_{01}=2.35\right)$, friend and stranger $(t(38)=2.12, p=.041$, $\left.d z=0.34, B F_{01}=1.002\right)$, partner and stranger $\left(t(38)=2.12, p=.040, d z=0.34, B F_{01}=1.002\right)$ were non-significant. However, the Bayes factors provide inconclusive evidence for either the $\mathrm{H} 0$ or $\mathrm{H} 1$ hypotheses for these three comparisons.

In the reward task, there was a main effect of Stimulus $(\mathrm{x} 9, \mathrm{x} 7, \mathrm{x} 5, \mathrm{x} 3, \mathrm{x} 1)(\mathrm{F}(4,152)=3.55$, $p=.008)$. There was also a linear trend in decreasing sensitivity with decreasing reward incentives $(\mathrm{F}(1,38)=7.15, p=.011)$. Post Hoc pairwise comparisons with Bonferroni corrections showed that sensitivity to x9 reward was significantly higher compared to $\mathrm{x} 3$ reward $\left(t(38)=3.31, p=.021, B F_{01}\right.$ $=0.074$ ). No other significant terms were found here (all $p s>.05$ ). However, the Bayes factors provided evidence for the null hypothesis for differences in sensitivity between $\mathrm{x} 9$ and $\mathrm{x} 7\left(B F_{01}=\right.$ 7.30), $x 7$ and $x 1\left(B F_{01}=3.22\right), x 5$ and $x 3\left(B F_{01}=5.04\right)$ and $x 5$ and $x 1\left(B F_{01}=7.06\right)$. There was no much evidence for the effects between $\mathrm{x} 9$ and $\mathrm{x} 5\left(B F_{01}=0.62\right), \mathrm{x} 9$ and $\mathrm{x} 1\left(B F_{01}=2.15\right), \mathrm{x} 7$ and $\mathrm{x} 5$ $\left(B F_{01}=1.92\right), x 7$ and $x 3\left(B F_{01}=0.29\right)$, and $x 3$ and $x 1\left(B F_{01}=2.79\right)$. 


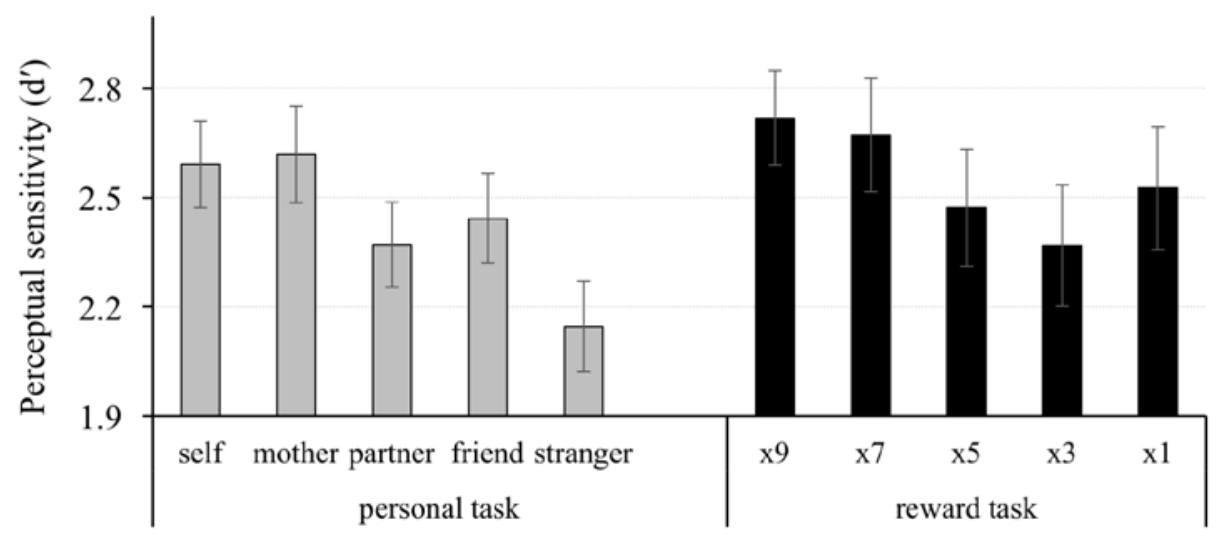

Fig. 6 Mean perceptual sensitivity index (d') for shapes associated with persons and reward values. The error bars represent SEM +/- 1 .

To test whether changes in perceptual sensitivity in the personal task (defined as $\left[\mathrm{d}^{\prime}{ }_{\text {stranger }}\right.$ $\left.d_{\text {self }}^{\prime}\right]$ ) could predict changes in perceptual sensitivity in the reward task (defined as $\left[d^{\prime}{ }_{x 1}^{-} d^{\prime}{ }_{x 9}\right]$ ), a regression analysis was carried out. The results showed that the changes in perceptual sensitivity in the personal task could not reliably predict the changes in perceptual sensitivity in the reward task (B $\left.=-0.057, \mathrm{SE}=0.18, t(38)=0.32, p=.75, d z=0.05, B F_{01}=7.63\right)$.

\section{Drift-diffusion computational results}

Decision bias (z/a). The decision bias parameter (or the relative starting point) was significantly higher for the reward task $(\mathrm{M}=0.58, \mathrm{SD}=0.09)$ compared to the personal task $(\mathrm{M}=$ $0.53, \mathrm{SD}=0.06)\left(t(38)=2.72, p=.01, d z=0.44, B F_{01}=0.29(\right.$ the inverse $\left.\mathrm{BF}=3.44)\right)$.

Drift rates $(v)$. The difference in drift rates between the reward task $(\mathrm{M}=2.82, \mathrm{SD}=1.33)$ and the personal task $(\mathrm{M}=2.58, \mathrm{SD}=1.06)$ did not reach significance $(t(38)=2.01, p=.052, d z=$ 0.32, $\left.B F_{01}=1.22\right)$. Because the Bayes factor was inconclusive here and p-value fell at the board of significance, we performed an additional analysis using an equivalence testing approach and the 'two-one-sided $t$-tests' (Lakens, 2017). In the ‘two-one-sided $t$-tests’ (TOST), an upper $(\Delta \mathrm{U})$ and lower $(-\Delta \mathrm{L})$ equivalence bounds are specified based on the smallest effect size of interest (e.g., a positive or negative difference of Cohen's $d=0.3$ ). Two composite null hypotheses are tested: H01: $\Delta \leq-\Delta \mathrm{L}$ and H02: $\Delta \geq \Delta \mathrm{U}$. When both these one-sided tests can be statistically rejected, one can conclude that $-\Delta \mathrm{L}<\Delta<\Delta \mathrm{U}$ (i.e., the observed effect falls within the equivalence bounds and is close enough to zero to be practically equivalent (Lakens, 2017). The TOST procedure indicated that 
the observed effect size was not significantly within the equivalence bonds of -0.3 and 0.3 scale points (or in Cohen's $d z$ : -0.16 and 0.16 ), $t(38)=-0.19, p=.42$.

To examine the effect of Stimulus on the drift rates in each task, two repeated measures ANOVAs were carried out.

In the personal task, there was a main effect of Stimulus $(F(4,152)=17.00, \mathrm{MSE}=13.074$, $p<.001$ ) (Figure 7, A) and a strong linear trend (a polynomial contrast, $\mathrm{F}(1,38)=47.83, p<.001$ ). Post Hoc pairwise comparisons with Bonferroni corrections showed that drift rates for self and mother were significantly larger compared to the rest of stimuli $\left(t(38)_{\text {self vs partner }}=5.78, p<.001, d z=\right.$ $0.93, B F_{01}=6.35 \mathrm{e}-05 ; t(38)_{\text {self vs friend }}=4.29, p=.001, d z=0.69, B F_{01}=0.005 ; t(38)_{\text {self vs stranger }}=$ 6.59, $p<.001, d z=0.89, B F_{01}=5.74 \mathrm{e}-06 ; t(38)_{\text {mother vs partner }}=4.37, p=.001, d z=0.69, B F_{01}=$ $\left.0.0042 ; t(38)_{\text {mother vs stranger }}=4.95, p<.001, d z=0.79, B F_{01}=0.0008\right)$. No other terms were significant here $\left(t(38)_{\text {partner vs friend }}=1.34, p=.65, d z=0.21, B F_{01}=3.39 ; t(38)_{\text {partner vs stranger }}=2.16, p=\right.$ $.37, d z=0.35, B F_{01}=0.93 ; t(38)_{\text {friend vs stranger }}=2.83, p=.075, d z=0.45, B F_{01}=0.23 ; t(38)_{\text {self vs mother }}=$ $\left.0.99, p=1.0, d z=0.16, B F_{01}=4.99 ; t(38)_{\text {mother vs friend }}=2.82, p=.08, d z=0.45, B F_{01}=0.23\right)$. However, the Bayes factors provided evidence for the effect in friend vs stranger and mother vs friend comparisons (the inverse $\mathrm{BF}=4.35$ for both comparisons).

In the reward task, there was a main effect of Stimulus $(\mathrm{F}(4,152)=6.73$, MSE $=6.85, p<$ .001) (Figure 7, A) and a strong quadratic trend (a polynomial contrast, $\mathrm{F}(1,38)=29.43, p<.001$ ). Post Hoc pairwise tests showed that drift rates for shapes associated with $\mathrm{x} 9$ reward were significantly larger compared to all intermediate reward values $\left(t(38)_{\times 9}\right.$ vs $\times 7=3.15, p=.032, d z=$ $0.50, B F_{01}=0.11 ; t(38)_{x 9 \text { vs } \times 5}=5.62, p<.001, d z=0.89, B F_{01}=0.0001 ; t(38)_{x 9 \text { vs } \times 3}=5.27, p<.001$, $\left.d z=0.84, B F_{01}=0.0003\right)$, but not to $\mathrm{x} 1$ reward $\left(t(38)_{\times 9}\right.$ vs $\left.\times 1=2.38, p=.22, d z=0.38, B F_{01}=0.61\right)$. No other significant terms were found here $\left(t(38)_{\mathrm{x} 7 \mathrm{vs} \times 5}=1.07, p=1.0, d z=0.17, B F_{01}=4.61\right.$; $t(38)_{\mathrm{x} 7 \mathrm{vs} \times 3}=1.42, p=1.0, d z=0.22, B F_{01}=3.06 ; t(38)_{\mathrm{x} 7 \mathrm{vs} \times 1}=0.35, p=1.0, d z=0.05, B F_{01}=7.55$; $t(38)_{\mathrm{x} 5 \mathrm{vs} \times 3}=0.43, p=1.0, d z=0.07, B F_{01}=7.33 ; t(38)_{\mathrm{x} 5 \mathrm{vs} x 1}=1.52, p=1.0, d z=0.24, B F_{01}=2.67$; $\left.t(38)_{\times 3 \text { vs } \times 1}=1.68, p=1.0, d z=0.27, B F_{01}=2.11\right)$. 

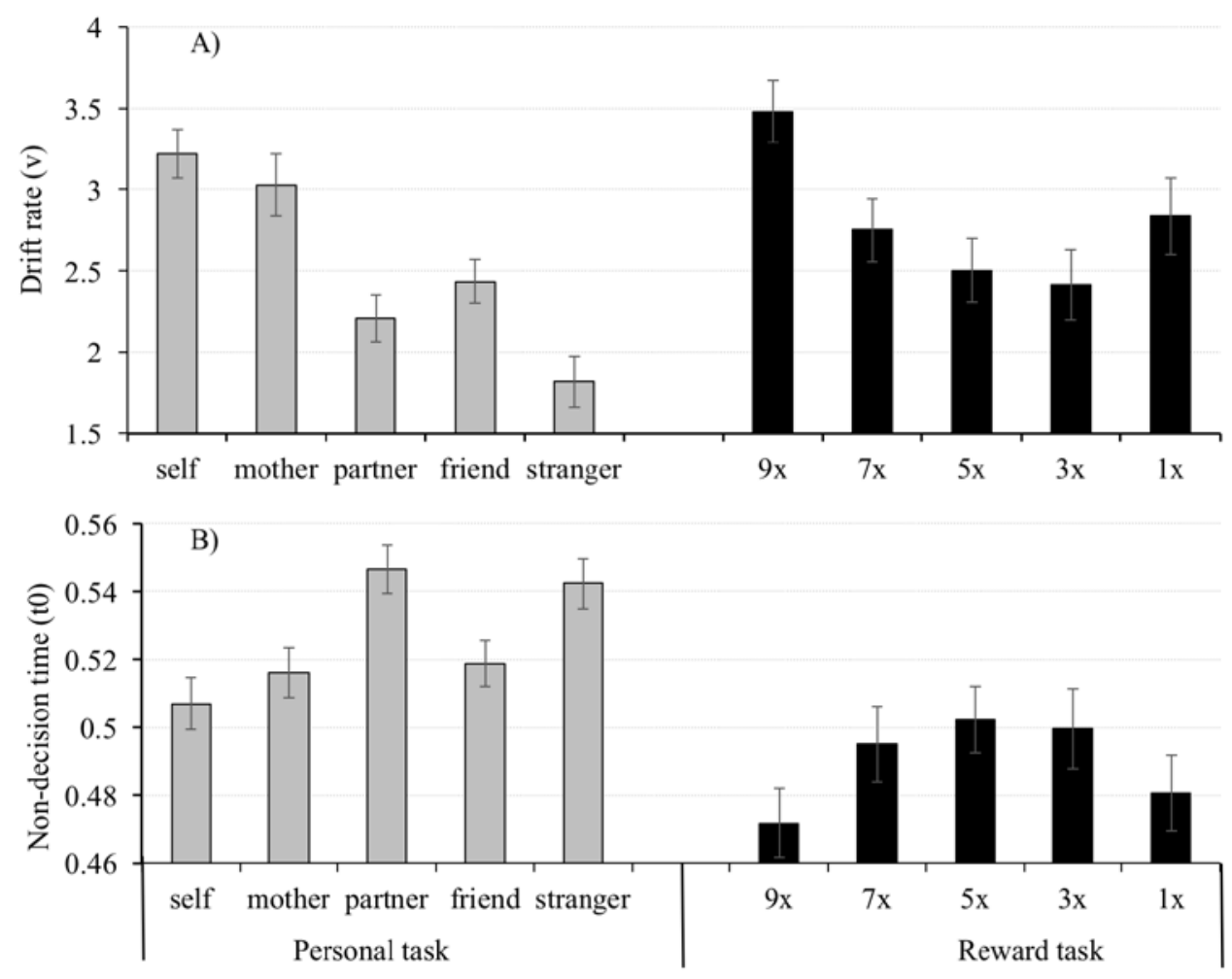

Fig. 7 Mean estimates of (A) drift rates $(v)$ and $(B)$ non-decision time $(t 0)$ in personal and reward tasks. The error bars represent SEM +/- 1 .

Non-decision time (t0). The non-decisional component was significantly longer in personal task $(\mathrm{M}=0.52, \mathrm{SD}=0.04)$ compared to reward task $(\mathrm{M}=0.49, \mathrm{SD}=0.06)(t(38)=3.65, p=.001$, $\left.d z=0.58, B F_{01}=0.03\right)$.

In the personal task, $(\mathrm{F}(4,152)=15.35, \mathrm{MSE}=.012, p<0.001)($ Figure $7, \mathrm{~B})$ the nondecisional time was significantly longer for partner and stranger compared to self, mother and friend $\left(t(38)_{\text {partner vs self }}=6.51, p<.001, d z=1.04, B F_{01}=7.29 \mathrm{e}-06\right.$; $t(38)_{\text {partner vs mother }}=5.45, p<.001, d z=$ $0.87, B F_{01}=0.0002 ; t(38)_{\text {partner vs friend }}=4.39, p<.001, d z=0.70, B F_{01}=0.004 ; t(38)_{\text {stranger vs self }}=4.83$, $p<.001, d z=0.87, B F_{01}=0.0011 ; t(38)_{\text {stranger vs mother }}=4.95, p<.001, d z=0.79, B F_{01}=0.0008$; $\left.t(38)_{\text {stranger vs friend }}=3.83, p<.005, d z=0.61, B F_{01}=0.019\right)$. No other pairwise comparisons reached significance level here $\left(t(38)_{\text {self vs mother }}=1.55, p=1.0, d z=0.25, B F_{01}=2.56 ; t(38)_{\text {self vs friend }}=1.75, p\right.$ $=.89, d z=0.28, B F_{01}=1.89 ; t(38)_{\text {friend vs mother }}=0.41, p=1.0, d z=0.06, B F_{01}=7.38 ; t(38)_{\text {stranger vs }}$ partner $\left.=0.65, p=1.0, d z=0.10, B F_{01}=6.53\right)$. 
In the reward task, there was a main effect of Stimulus $(\mathrm{F}(4,152)=10.12$, MSE $=.007, p<$ 0.001 ) and a strong quadratic trend (a polynomial contrast, $\mathrm{F}(1,38)=28.59, p<.001$ ). Both $\mathrm{x} 9$ and x1 reward values showed significantly shorted non-decision time compared to intermediate rewards $\left(t(38)_{\times 9}\right.$ vs $\times 7=3.82, p=.005, d z=0.61, B F_{01}=0.019 ; t(38)_{x 9 \text { vs } \times 5}=5.04, p<.001, d z=0.81, B F_{01}=$ $0.006 ; t(38)_{x 9}$ vs $\times 3=4.25, p<.001, d z=0.68, B F_{01}=0.006 ; t(38)_{x 1 \text { vs } \times 3}=3.59, p=.009, d z=0.57$, $\left.B F_{01}=0.035 ; t(38)_{x 1 \text { vs } x 5}=3.45, p=.014, d z=0.55, B F_{01}=0.051\right)$. The difference between $x 1$ and x7 rewards did not reach significance level, and a Bayes factor provided weak evidence for either the null and the alternative hypotheses $\left(t(38)_{\mathrm{x} 1 \mathrm{vs} \times 7}=2.45, p=.19, d z=0.39, B F_{01}=0.52\right)$. The differences in non-decision time between intermediate reward values were non significant $\left(t(38)_{x 7} \mathrm{vs}\right.$ ${ }_{\mathrm{x} 5}=1.31, p=1.0, d z=0.21, B F_{01}=3.53 ; t(38)_{\mathrm{x} 7 \mathrm{vs} \mathrm{x} 3}=0.85, p=1.0, d z=0.14, B F_{01}=5.65 ; t(38)_{\mathrm{x} 5 \mathrm{vs}}$ $\left.\mathrm{x} 3=0.52, p<.005, d z=0.08, B F_{01}=7.03\right)$. There was also no significant difference between $\mathrm{x} 9$ and $\mathrm{x} 1$ rewards $\left(t(38)_{\mathrm{x} 1 \text { vs } \mathrm{x}}=1.54, p=.14, d z=0.24\right)$ and the Bayes factor was inconclusive here $\left(B F_{01}=\right.$ 2.59).

We assessed goodness of fit (Voss \& Voss, 2007; Voss et al., 2015) for 39 individual models in each task. In the personal task, the model showed bad fit to the data for two participants (KS, $p=.006, p=.024)$. In the reward task, the model fit test was significant for four participants ( $p=$ $.012, p=.003, p=.048, p=.004)$. A binomial test indicated that the proportion of models with good fit to the data $(0.948,[95 \% \mathrm{CI}[0.83,0.99])$ in the personal task and $(0.0 .897,[95 \% \mathrm{CI}[0.76,0.97])$ in the reward task was significantly higher then the proportion of models with bad fit ( $p s<.001,2-$ sided). These results suggest that the individual models describe the individual RT distributions well.

The relationship between subjective experience of the personal distance and RT difference

Individual indices of the personal distance between the participant and others (calculated as indices of the relative personal distance between self and stranger scaled by the distance between self and mother (self and partner, self and friend)) were entered in to a repeated measures ANOVA to examine the effect of person (mother, partner, friend) on the relative personal distance.

There was a main effect of person $(\mathrm{F}(2,76)=19.92, \mathrm{MSE}=12,956, p<0.001)$ (Figure 8) suggesting larger personal distance to friend compared to mother $(t(38)=4.86, p<0.001, d z=0.78$, 
$\left.B F_{01}=0.001\right)$ and partner $\left(t(38)=4.16, p<0.001, d z=0.66, B F_{01}=0.007\right)$. The difference between the distance to mother and to partner did not rich significance level $(t(38)=2.48, p=0.061, d z=$ 0.39), and the Bayes factor was inconclusive here $\left(B F_{01}=0.49\right)$.

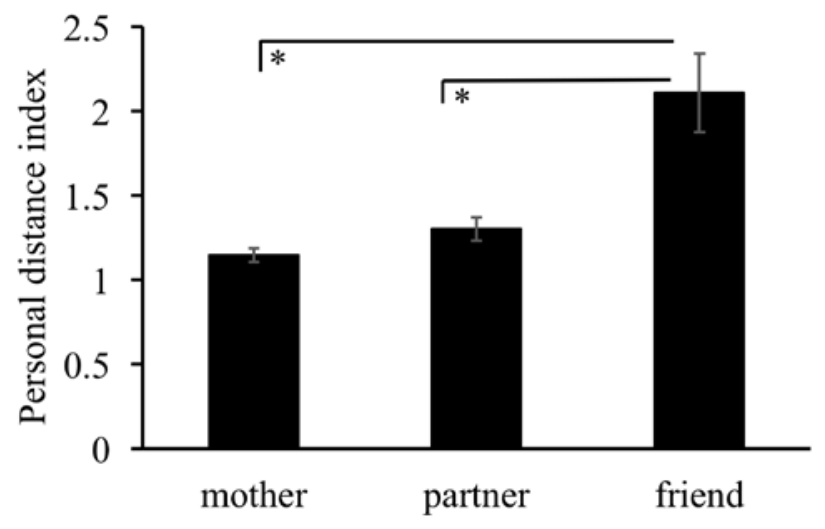

Fig. 8 Personal distance indices represent relative distance from self to mother (calculated as [self stranger]/[self-mother]), partner ([self - stranger]/[self-partner]) and friend (self-stranger]/[selfpartner]). Error bars represent +/-SEM. The asterisks indicate significant differences between conditions (adjusted for multiple comparisons using Bonferroni correction).

Two simple linear regressions were carried out to test whether the indices of the personal distance between self and mother and mother and partner could predict the difference in RT between them. The results suggested that a significant proportion of the total variation in the RT difference between self and mother was predicted by the index of the personal distance between self and mother (Table 5, Figure 8). The coefficient of determination $\left(\mathrm{R}^{2}\right)$ indicated that approximately 32\% and $37 \%$ of the variation in the RT difference was predicted by the index of the personal distance to mother and partner respectively. According to Cohen’s effect size (Cohen, 1988), this refers to substantial effect. Similar results were found for self-partner relationship (Table 4, Figure 8). In contrast, the index of the personal distance between self and friend could not reliably predict the difference in RT between self and friend $\left(\mathrm{BF}_{01}=1.57\right)$ (Table 4).

Table 4 Results of linear regression analyses between indices of the personal distance and RT differences

\begin{tabular}{llllllll}
\hline Independent & Dependent & \multicolumn{3}{c}{ ANOVA } & \multirow{2}{*}{$\mathrm{R}^{2}$} & $\beta$ & $p$ \\
\cline { 2 - 4 } variable & variable & $\mathrm{F}(1,38)$ & MSE & $p$ & & & \\
\hline self-mother & RT difference & 17.41 & .811 & $<0.001$ & 0.32 & 0.56 & $<0.001$
\end{tabular}




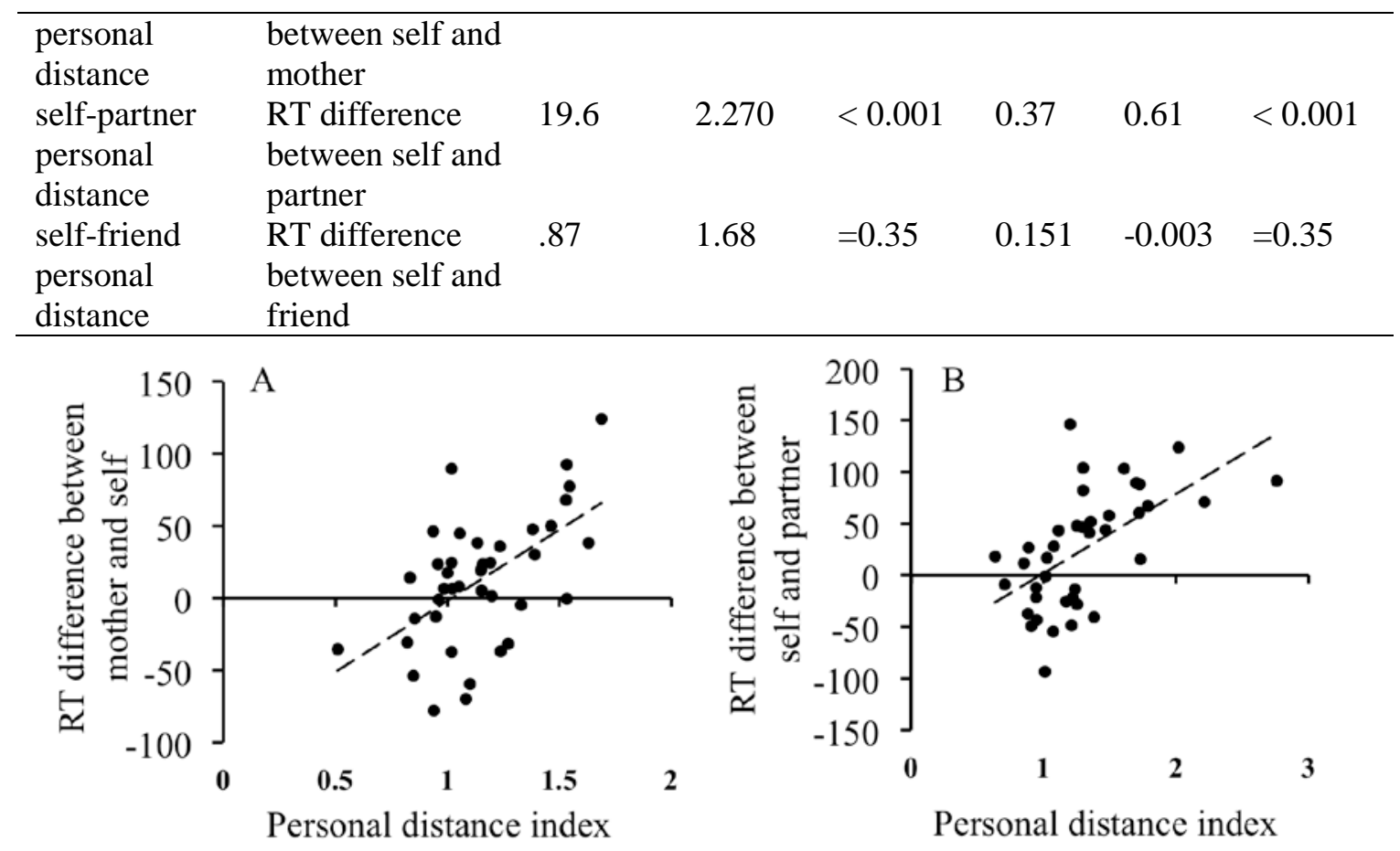

Fig. 8 A linear regression plot between the index of personal distance and RT difference for selfmother (A) and self-partner (B) relationship.

\section{General Discussion}

The experiment provides evidence for common and distinct effects of personal relevance and reward values on perceptual decision process. The evidence is summarised in Table 5.

Table 5 Common and distinct effects of personal relevance and reward values on perceptual decision process in speeded two-alternative choice matching task. BF* indicates that the Bayes factor provides inconclusive evidence for the result.

\begin{tabular}{l}
\hline \multicolumn{1}{c}{ Personal relevance } \\
\hline Facilitation effects of personal relevance/reward value on accuracy performance (accuracy rates increase \\
linearly with increasing personal relevance/reward value in matched trials). No effect of personal \\
relevance/reward value on accuracy in mismatched trials. \\
Facilitation effects of personal relevance/reward value on RT performance in matched and mismatched \\
trials. \\
No effect of Task (personal, reward) on perceptual sensitivity (d'). A trend in decreasing perceptual \\
sensitivity with decreasing personal relevance/reward incentives. \\
No effect of Task on the the average rate of information uptake in decision process (drift rates, v) (BF*). \\
\hline Distinct effects \\
\hline Slower responses to shape-person associations Faster responses to shape-reward associations
\end{tabular}


compared to shape-reward associations

RT increases linearly with increasing personal relevance of stimuli. This effect is strong and holds for matched and mismatched pairings.

All personal associations excluding associations with partner showed random effects

distinguishable from zero in matched pairs

RT-gains for self, mother and partner in matched and mismatched trials

Greater RT-gains for self and mother (compared to partner and friend).

No difference in RT-gains between self and mother $\left({ }^{*} \mathrm{BF}\right)$ and between partner and friend $(* \mathrm{BF})$

Drift rates in decision process increase linearly with increasing personal relevance associated with stimuli (across the continuum: stranger-friendpartner-mother-self)

Longer non-decisional time in perceptual decision processes compared to shape-reward associations. Shapes associated with partner and stranger showed the longest non-decisional times.

The decision bias parameter (z/a) is lower

Associations with self serve as an anchor point in the personal relevance continuum (self-motherpartner-friend-stranger) compared to shape-person associations

U-shape relationship between reward value and RT. This effect holds for matching pairings only.

Prediction intervals on the random effects of $x 1, x 7$ and marginally $\mathrm{x} 5$ reward associations overlap zero in matched pairs

RT gain for the $\mathrm{x} 9$ reward value only $(* \mathrm{BF})$ in matched trials. No RT-gain in mismatched trials

Response bias is more liberal compared to personal task

Drift rates in decision process conform to a

U-shape where the highest speed reflects the highest and the lowest reward values (across the continuum (x9-x7-x5-x3-x1) reward)

Shorter non-decisional time in perceptual decision processes compared to shape-person associations. The changes in non-decision time conform to an inverted U-shape where the shortest time reflects the highest and the lowest reward values

The decision bias parameter (z/a) is higher

No evidence that associations with the highest reward value serve as an anchor point in the reward relevance continuum (x9-x7-x5-x3-x1)

\section{Common effects of personal relevance and reward values}

Our finding that personal relevance and reward modulate response speed and accuracy adds to the mounting evidence of self- and reward- prioritization effects reported in cognitive research (Dambacher \& Hubner, 2013; Karsilar, Simen, Papadakis, \& Balci, 2014; Madan \& Spetch, 2012; Sui \& Humphreys, 2015b, 2015c; Sui et al., 2015). More importantly, this finding confirms previous results that linking neutral objects with personal relevance and reward values enhances perceptual matching (Sui et al., 2012). For example, the current study's findings are similar to results in Sui, et al., (2012) who reported faster responding for self and high reward associations, and suggested no qualitative differences between the two effects on behavioural performance. 
The common effects of personal relevance and reward values here were also evident in perceptual sensitivity data. There was no difference in sensitivity between the tasks. Furthermore, sensitivity index for shapes associated with high personal relevance (self and mother) and reward value (9x) was significantly larger across conditions in each task. Since all stimuli in our study were of high contrast, we suggest that these changes in $\mathrm{d}^{\prime}$ imply stronger representations and/or activation of stored representation for personally and reward important associations. As a result, more robust representations change perceptual processing of the items in a bottom-up fashion generating a stronger perceptual signal (Wildegger, Riddoch, \& Humphreys, 2015). Importantly, the changes in sensitivity for self-association did not relate to changes in sensitivity for high reward (9x) indicating qualitative differences in their representations.

Thus, our data supports the finding that the effects of prioritizing personal and rewardrelated information mimic each other, engendering similar performance on the tasks (Northoff \& Hayes, 2011; Sui et al., 2012). The question here is how this similarity arises?

The similarity between the effects of personal relevance and reward values may imply common cognitive and neural mechanisms governing the effects. Northoff and colleagues proposed that cortical and subcortical structures associated with self and reward processing are mediated by resting state activity (Northoff, 2016), which predicts the degree of reward-related activity (Duncan et al., 2013) and self-related activity (Lane et al., 2016). Although this account has an important application in understanding how different brain structures interact to consolidate high cognitive functions (including reward and self-referential), it does not shed light on the differential effects of personal relevance and reward values. Furthermore, there is evidence that the similarity between the effects is not unique. For example, 'tagging’ a neutral shape with emotional information (e.g., positive emotion vs. neutral emotion) (Stolte, Humphreys, Yankouskaya, \& Sui, 2017) or with team membership relevance (e.g., in-group vs. out-group) (Moradi, Sui, Hewstone, \& Humphreys, 2015) results in behavioural patterns very similar to those observed in the personal and reward tasks in the current study. Moreover, self-, reward- and in-group biases showed similar modulatory effects on perception by increasing processing efficiency during information processing (Yankouskaya et al., 2017). The evidence points to more generic mechanisms underlying the effects of social biases. 
Recent progress has been made toward exploring neurobiological (Groppe et al., 2013) and neurocognitive accounts (Humphreys \& Sui, 2016). Alternatively, 'tagging' a neutral object with socially related information may trigger activation of the salience network which coordinates behavioural responses to prioritize the most important signals (Medford \& Critchley, 2010). However, it is still largely unknown whether the biases occur as multiple modalities of the same cognitive processes, and how they relate to each other in the brain.

\section{Distinct effects of personal relevance and reward values}

Relative to personal associations, responses to shapes associated with reward were significantly faster. This was not due to changes in perceptual sensitivity and, therefore, it is unlikely that enhanced responding in reward task occurs at early perceptual level. These findings indicate that the difference in RT between the tasks may reflect longer non-decisional component or/and stimulus properties in each task. Indeed, the drift-diffusion analysis provides strong support for the assumption that the difference between processing of personal and reward associations reflects longer non-decisional time in personal task. Studies employing the drift-diffusion approach suggest that longer duration of the non-decisional process during perceptual categorization engages additional cognitive resources (e.g., attention networks, working memory capacity, assessing the semantic meaning of a stimulus) that cause slower encoding or response execution (Ratcliff \& McKoon, 2008). The shapes 'tagged' with personal relevance have 'richer' representations compared to shapes 'tagged' with reward values because it reflects the content associated with the meaning of the 'tag'. Previous studies using the perceptual matching procedure with tree-item personal associations and three-items reward associations reported no significant differences in response time between personal and reward tasks (Sui et al., 2012; Sui \& Humphreys, 2015b; Sui et al., 2016). In our study, we used five-items associations in each task that exerts higher cognitive demands on working memory. As a result, retrieving information from memory about personal associations may require additional time compared to information about reward associations.

Importantly, the effect of Task was preserved even when we took into account by-subject and by-stimulus variability in the data. The goodness of fit of the full model which included all random effects significantly decreased when one of the random effects was removed. This finding 
suggests that the Task factor has a systematic and predictable influence on our data and provides strong evidence against the assumption that 'our self is nothing but reward' (Northoff \& Hayes, 2011). Note that the experimental design, procedures and instructions to the participants were identical in the personal and reward tasks and therefore the effects of task cannot be attributed to methodological issues.

Exploring the random effect of stimulus revealed that shapes associated with self and mother reliably contribute to random effects. Assuming that the self serves as an anchor point in the continuum of personal relevance, it is not surprising that associations with others do not show a nonsystematic influence on response times. The plausibility of this assumption is supported by the fact that this effect of self-associations is observed in matched and mismatched trials. The finding that both self and mother showed idiosyncratic influence on the data may reflect the intention of young adults to be independent of parenteral influence on one hand but closeness and attachment on the other hand. If associations with the highest reward value would serve as an anchor point, we should expect to see similar effects for stimuli in reward task. However, our data do not support this assumption.

We predicted that response speed in both tasks should linearly decrease from shapes associated with a higher value (e.g., self and 9x reward) to lower value (e.g., stranger, $£ 1$ ). In contrast to our hypothesis, we found a strong linear trend in personal, but not in reward task where the difference between the highest and the lowest reward values was diminished. This trend was also evident in drift rates and non-decisional time measures. Previous studies reported biased memory for the highest and the lowest outcomes associated with reward (Klingberg, 2010; Madan \& Spetch, 2012) and more recent research linked the memory bias for reward with decision making (Madan et al., 2014; Wimmer \& Buchel, 2016). For example, Madan et al. (2014) argued that the memory bias from the past experience results in overweighting the largest gains and largest losses, leading people to seek for relative gains rather than relative losses. Furthermore, in a task where monetary reward anticipation was linked to incidental objects, neural patterns related to reward experiences were reexpressed on later exposure to stimuli and reactivation strongly correlated with the behavioral performance (Wimmer \& Buchel, 2016). In contrast, personal relevance is considered as a system 
where 'the self' is prima facie relatively to others (Abraham, 2013; Sui, 2016; Sui \& Humphreys, 2015a) and memory bias increases with increasing personal relevance to self (Cunningham, Turk, Macdonald, \& Neil Macrae, 2008; Macrae, Moran, Heatherton, Banfield, \& Kelley, 2004; Turk et al., 2013).

Earlier research reported a greater facilitation effect (RT-gain) in the personal task compared to the reward task (Sui \& Humphreys, 2015c; Sui et al., 2015) . It was suggested that selfassociations uniquely enhance the binding of information at both perceptual and conceptual levels while high-reward-associations led to gains at a conceptual level; but these stimuli had weaker processing capacity. Although our data showed similar results, we must be cautious in the interpretation of these findings. The magnitude of RT-gains in reward task may be limited by relatively small differences in reward values (i.e., £8, £6, £4, £2). Particularly, in our study, only the difference between $9 \mathrm{x}$ and $1 \mathrm{x}$ values (£8) generated significant RT-gains, whereas smaller amount (£6, $£ 4, £ 2)$ failed to generate any RT-gain.

We did not find evidence for the relationship between RT-gains for self and high-reward value (9x). Sui and Humphreys (2015c) argued that the lack of correlation between these values implied separate origin of self and reward biases that operate as stable, trait-like behaviour. Our findings support this interpretation, however, individual differences in past experience with reward values may add a great variability to the data.

Although our data support the explanation of slower RT in performing a personal task, the question about the strength of personal vs reward representations calls for further research. For example, a recent study using an associative matching procedure found that even extended learning undertaken to equate memory to various identity-based associations, did not eliminate the effects of self-prioritization, and thus, leaving the question open as to whether the differences between self and others are cognitive or perceptual in nature (Reuther \& Chakravarthi, 2016). If, presumably, the facilitation effects of self and high reward values here arises from potential memory differences introduced during the formation of associations, we should find no difference in the drift rates, but larger non-decision time in performing the personal task than in the reward task. Although inferential statistics in our study support this assumption, the Bayes factor does not provide enough 
evidence for the null hypothesis in the drift rates difference between the task. Furthermore, the result of equivalence testing does not support the absence of the effect. Therefore, it is unlikely that the facilitation effects of self and high reward emerges as a result of memory differences only. Manipulating low properties of the stimuli in each task and examining their effects on the parameters of diffusion process may help to separate perceptual from cognitive processes.

\section{The relationship between personal distance and RT-gains}

We employed a projective technique aiming to elicit subjective projections from self to others and assess an individual's feelings of personal distance to close and familiar people. The data indicated that participants placed themselves closer to mother and partner, and the degree of their remoteness to them predicted the magnitude of RT-gains (i.e. RT advantage for mother and partner compared to a stranger). Moreover, responses to shapes associated with mother and partner were significantly faster compared to friend - the results that were more evident in faster drift rates and shorter non-decision time. These findings suggest that a closer relationship with a person, compared to a more distant relationship, results in stronger representations about attributes associated with the individual and facilitates perceptual decision making.

Interestingly, although a large body of research emphasizes a significant role of friends in social, personal and academic life in young adults (Collins \& Laursen, 2004), our data indicate a larger personal distance to a friend (compared to mother and partner) that showed no systematic relationship with the facilitation in RT performance. This finding is in line with studies suggesting that when emerging young adults are involved in a romantic relationship, the importance of the role of friends in their happiness is less pronounced or not pronounced at all (Demir \& Özdemir, 2010; Lucas, Dyrenforth, Vohs, \& Finkel, 2006; Whisman, Sheldon, \& Goering, 2000).

The main reason for using the projective technique here was to bridge personal projections to others and RT biases in personal perception. We suggest that the perceptual matching procedure may have an important application in interpersonal research by informing and supporting qualitative methods.

\section{Conclusion}


Personal relevance and monetary reward generate common and differential effects on perceptual decision making. The common effects are manifested in the facilitation of behavioural performance for high personal relevance and high reward value as socially important signals. The differential effects reflect non-decisional time, and task-specific prioritization of stimuli. Our findings support the parallel processing model (Northoff \& Hayes, 2011) and suggest that selfspecific processing occurs in parallel with high-reward processing.

\section{Limitations and directions for further research}

The present study focused on one type of reward (monetary). Considering that other types of reward (e.g., facial attractiveness, social approval) may have a different impact on individual experience, it is important to examine whether the relationship between different types of reward and self-relevance yields the same characteristics. By empirically testing the fields of self and reward we will gain a better understanding of how each concept emerges.

We do not exclude the possibility that personal and reward relevance are liable for interactions at higher cognitive level where the interactions may be triggered by different factors or linked to each other experimentally. Exploring key factors triggering the interactions is an important area of the research in social cognitive science that requires developing a new methodological approach.

Finally, this is the first study that attempted to highlight commonalities and differences of the effects of personal and reward relevance on perceptual decision making. We hope that the results reported here will provide a strong starting-point for further research using the drift-diffusion modelling.

\section{Acknowledgements}

The work was supported by grants from the European Research Council (Pepe: 323883, 2013) to Professor Glyn W. Humphreys ${ }^{7}$

\section{Compliance with Ethical Standards}

All procedures performed in studies involving human participants were in accordance with the ethical standards of the institutional and/or national research committee and with the 1964 Helsinki declaration and its later amendments or comparable ethical standards. Informed consent was

\footnotetext{
${ }^{7}$ Professor G. W. Humphreys passed away after the data were collected
} 
obtained from all individual participants included in the study. Informed consent was obtained from all individual participants included in the study.

\section{Conflict of Interest}

Author A. Yankouskaya declares that she has no conflict of interest.

Co-Author M.Stolte declares that he has no conflict of interest.

Co-Author R. Buhrle declares that he has no conflict of interest.

Co-Author E.Lugt declares that she has no conflict of interest.

Co-Author J.Sui declares that she has no conflict of interest.

\section{References}

Abraham, A. (2013). The world according to me: personal relevance and the medial prefrontal cortex. Frontiers in Human Neuroscience, 7, 341. doi:10.3389/fnhum.2013.00341

Bai, Y., Nakao, T., Xu, J., Qin, P., Chaves, P., Heinzel, A., . . Northoff, G. (2016). Resting state glutamate predicts elevated pre-stimulus alpha during self-relatedness: A combined EEGMRS study on "rest-self overlap". Social Neuroscience, 11(3), 249-263. doi:10.1080/17470919.2015.1072582

Baayen, R.H., Davidson, D.J., Bates, D.M. (2008). Mixed-effects modeling with crossed random effects for subjects and items. Journal of Memory and Language, 59, 390-412.

Chakraborty, A. \& Chakrabarti, B. (2015). Is it me? Self-recognition bias across sensory modalities and its relationship to autistic traits. Molecular Autism, 6 (1). 20. DOI: 10.1186/s13229-0150016-1

Christoff, K., Cosmelli, D., Legrand, D., \& Thompson, E. (2011). Specifying the self for cognitive neuroscience. Trends in Cognitive Sciences, 15(3), 104-112. doi:10.1016/j.tics.2011.01.001

Clithero, J. A., \& Rangel, A. (2014). Informatic parcellation of the network involved in the computation of subjective value. Social Cognitive and Affective Neuroscience, 9(9), 12891302. doi:10.1093/scan/nst106 
Cohen, J. (1988). Statistical power analysis for the behavioral sciences (2nd ed.). Hillsdale, NJ: Erlbaum.

Collins, W. A., \& Laursen, B. (2004). Changing Relationships, Changing Youth. The Journal of Early Adolescence, 24(1), 55-62. doi:DOI: 10.1177/0272431603260882

Conway, M. A., Pothos, E. M., \& Turk, D. J. (2016). The self-relevance system? Cognitive Neuroscience, 7(1-4), 20-21. doi:10.1080/17588928.2015.1075484

Corrigendum: Signal Detection Measures Cannot Distinguish Perceptual Biases From Response Biases. (2016). Perception, 45(8), 964-965. doi:10.1177/0301006616637983

Cunningham, S. J., Turk, D. J., Macdonald, L. M., \& Neil Macrae, C. (2008). Yours or mine? Ownership and memory. Conscious and Cognition, 17(1), 312-318. doi:10.1016/j.concog.2007.04.003

D'Argembeau, A. (2013). On the role of the ventromedial prefrontal cortex in self-processing: the valuation hypothesis. Frontiers in Human Neuroscience, 7, 372. doi:10.3389/fnhum.2013.00372

Dambacher, M., \& Hubner, R. (2013). Investigating the speed-accuracy trade-off: better use deadlines or response signals? Behavior Research Methods, 45(3), 702-717. doi:10.3758/s13428-012-0303-0

Demir, M., \& Özdemir, M. (2010). Friendship, Need Satisfaction and Happiness. Journal of Happiness Studies, 11, 234. doi:doi:10.1007/s10902-009-9138-5

Denny, B. T., Kober, H., Wager, T. D., \& Ochsner, K. N. (2012). A meta-analysis of functional neuroimaging studies of self- and other judgments reveals a spatial gradient for mentalizing in medial prefrontal cortex. Journal of Cognitive Neuroscience, 24(8), 1742-1752. doi:10.1162/jocn_a_00233

Doering, S., Enzi, B., Faber, C., Hinrichs, J., Bahmer, J., \& Northoff, G. (2012). Personality functioning and the cortical midline structures--an exploratory FMRI study. PLoS One, 7(11), e49956. doi:10.1371/journal.pone.0049956

Duncan, N. W., Wiebking, C., Tiret, B., Marjanska, M., Hayes, D. J., Lyttleton, O., . . Northoff, G. (2013). Glutamate concentration in the medial prefrontal cortex predicts resting-state 
cortical-subcortical functional connectivity in humans. PLoS One, 8(4), e60312. doi:10.1371/journal.pone.0060312

Enzi, B., de Greck, M., Prosch, U., Tempelmann, C., \& Northoff, G. (2009). Is our self nothing but reward? Neuronal overlap and distinction between reward and personal relevance and its relation to human personality. PLoS One, 4(12), e8429. doi:10.1371/journal.pone.0008429

Farinelli, M., Panksepp, J., Gestieri, L., Leo, M.R., Agati, R., Maffei, M.,...Northoff, G. (2013). SEEKING and depression in stroke patients: An exploratory study. Journal of Clinical and Experimental Neuropsychology, 35(4)., 348-358. doi: 10.1080/13803395.2013.776009

Fossati, P., Hevenor, S. J., Lepage, M., Graham, S. J., Grady, C., Keightley, M. L., . . Mayberg, H. (2004). Distributed self in episodic memory: neural correlates of successful retrieval of selfencoded positive and negative personality traits. Neuroimage, 22(4), 1596-1604. doi:10.1016/j.neuroimage.2004.03.034

Gallagher, S. (2000). Philosophical conceptions of the self: Implications for cognitive science. Trends in Cognitive Sciences, 4(1), 14-21.

Goldfarb, S., Leonard, N. E., Simen, P., Caicedo-Nunez, C. H., \& Holmes, P. (2014). A comparative study of drift diffusion and linear ballistic accumulator models in a reward maximization perceptual choice task. Frontiers in Neuroscience, 8, 148. doi:10.3389/fnins.2014.00148

Groppe, S. E., Gossen, A., Rademacher, L., Hahn, A., Westphal, L., Grunder, G., \& Spreckelmeyer, K. N. (2013). Oxytocin influences processing of socially relevant cues in the ventral tegmental area of the human brain. Biological Psychiatry, 74(3), 172-179. doi:10.1016/j.biopsych.2012.12.023

Humphreys, G. W., \& Sui, J. (2016). Attentional control and the self: The Self-Attention Network (SAN). Cognitive Neuroscience, 7(1-4), 5-17. doi:10.1080/17588928.2015.1044427

Karsilar, H., Simen, P., Papadakis, S., \& Balci, F. (2014). Speed accuracy trade-off under response deadlines. Frontiers in Neuroscience, 8, 248. doi:10.3389/fnins.2014.00248

Kelley, W. M., Macrae, C. N., Wyland, C. L., Caglar, S., Inati, S., \& Heatherton, T. F. (2002). Finding the self? An event-related fMRI study. Journal of Cognitive Neuroscience, 14(5), 785-794. doi:10.1162/08989290260138672 
Klingberg, T. (2010). Training and plasticity of working memory. Trends in Cognitive Science, 14(7), 317-324. doi:10.1016/j.tics.2010.05.002

Lakens, D. (2013). Calculating and reporting effect sizes to facilitate cumulative science: a practical primer for t-tests and ANOVAs. Frontiers in Psychology, 4, 863. doi:10.3389/fpsyg.2013.00863

Lakens, D. (2017). A Practical Primer for t Tests, Correlations, and Meta-Analyses. Social Psychological and Personality Science, 8(4), 355-362. doi.org/10.1177/1948550617697177

Lane, T., Duncan, N. W., Cheng, T., \& Northoff, G. (2016). The Trajectory of Self. Trends in Cognitive Science, 20(7), 481-482. doi:10.1016/j.tics.2016.03.004

Lucas, R. E., Dyrenforth, P. S., Vohs, K. D., \& Finkel, E. J. (2006). Does the Existence of Social Relationships Matter for Subjective Well-Being? In K. D. Vohs \& E. J. Finkel (Eds.), Self and relationships: Connecting intrapersonal and interpersonal processes (pp. 254-273). New York, NY, US:: Guilford Press, xv,.

Macmillan, N. A., \& Creelman, C. D. (1991). Detection Theory: A User’s Guide. New York: Cambridge University Press.

Macrae, C. N., Moran, J. M., Heatherton, T. F., Banfield, J. F., \& Kelley, W. M. (2004). Medial prefrontal activity predicts memory for self. Cerebral Cortex, 14(6), 647-654. doi:10.1093/cercor/bhh025

Madan, C. R., Ludvig, E. A., \& Spetch, M. L. (2014). Remembering the best and worst of times: memories for extreme outcomes bias risky decisions. Psychonomic Bulletin \& Review, 21(3), 629-636. doi:10.3758/s13423-013-0542-9

Madan, C. R., \& Spetch, M. L. (2012). Is the enhancement of memory due to reward driven by value or salience? Acta Psychologica (Amst), 139(2), 343-349. doi:10.1016/j.actpsy.2011.12.010

Maresh, E., Allen, J., \& Coan, J. (2014). Increased default mode network activity in socially anxious individuals during reward processing. Biology of Mood \& Anxiety Disorders, 4:7. DOI: $10.1186 / 2045-5380-4-7$ 
Medford, N., \& Critchley, H. D. (2010). Conjoint activity of anterior insular and anterior cingulate cortex: awareness and response. Brain Structure and Functions, 214(5-6), 535-549. doi:10.1007/s00429-010-0265-x

Moradi, Z., Sui, J., Hewstone, M., \& Humphreys, G. W. (2015). In-group modulation of perceptual matching. Psychonomic Bulletin Review, 22(5), 1255-1277. doi:10.3758/s13423-014-07988.

Morey, R. D., Rouder, J. N., Pratte, M. S., \& Speckman, P. L. (2011). Using MCMC chain outputs to efficiently estimate Bayes factors. Journal of Mathematical Psychology, 55, 368-378

Moustafa, A. A., Keri, S., Somlai, Z., Balsdon, T., Frydecka, D., Misiak, B., \& White, C. (2015). Drift diffusion model of reward and punishment learning in schizophrenia: Modeling and experimental data. Behavioral Brain Research, 291, 147-154. doi:10.1016/j.bbr.2015.05.024

Northoff, G. (2016). Is the self a higher-order or fundamental function of the brain? The "basis model of self-specificity" and its encoding by the brain's spontaneous activity. Cognitive Neuroscience, 1(4), 203-222. doi:doi: 10.1080/17588928.2015.1111868

Northoff, G., \& Bermpohl, F. (2004). Cortical midline structures and the self. Trends in Cognitive Science, 8(3), 102-107. doi:10.1016/j.tics.2004.01.004

Northoff, G., \& Hayes, D. J. (2011). Is our self nothing but reward? Biological Psychiatry, 69(11), 1019-1025. doi:10.1016/j.biopsych.2010.12.014

R Core Team (2017). R: A language and environment for statistical computing. R Foundation for Statistical Computing, Vienna, Austria. URL https://www.R-project.org/. Ratcliff, R. (2015). Modeling one-choice and two-choice driving tasks. Attention, Perception \& Psychophysics, 77(6), 2134-2144. doi:10.3758/s13414-015-0911-8

Ratcliff, R., \& McKoon, G. (2008). The diffusion decision model: theory and data for two-choice decision tasks. Neural Comput, 20(4), 873-922. doi:10.1162/neco.2008.12-06-420

Ratcliff, R., Smith, P. L., Brown, S. D., \& McKoon, G. (2016). Diffusion Decision Model: Current Issues and History. Trends in Cognitive Science, 20(4), 260-281. doi:10.1016/j.tics.2016.01.007 
Rouder, J. N., Speckman, P. L., Sun, D., Morey, R. D., \& Iverson, G. (2009). Bayesian $t$ tests for accepting and rejecting the null hypothesis. Psychonomic Bulletin \& Review, 16, 225-237.

Rushworth, M. F., Noonan, M. P., Boorman, E. D., Walton, M. E., \& Behrens, T. E. (2011). Frontal cortex and reward-guided learning and decision-making. Neuron, 70(6), 1054-1069. doi:10.1016/j.neuron.2011.05.014

Schneider, F., Bermpohl, F., Heinzel, A., Rotte, M., Walter, M., Tempelmann, C., . . . Northoff, G. (2008). The resting brain and our self: self-relatedness modulates resting state neural activity in cortical midline structures. Neuroscience, 157(1), 120-131. doi:10.1016/j.neuroscience.2008.08.014

Scott-Van Zeeland, A.A., Dapretto, M., Ghahremani, D.G., Poldrack, R.A., \& Bookheimer, S.Y. (2010). Reward processing in Autism. Autism research, 3(2), 53-67. doi: 10.1002/aur.122. Stanislaw, H., \& Todorov, N. (1999). Calculation of signal detection theory measures. Behavior Research Methods, Instruments, \& Computers, 31(1), 137-149.

Stolte, M., Humphreys, G., Yankouskaya, A., \& Sui, J. (2017). Dissociating biases towards the self and positive emotion. Quarterly Journal of Experimental Psychology (Hove), 70(6), 10111022. doi:10.1080/17470218.2015.1101477

Sui, J. (2016). Self-Reference Acts as a Golden Thread in Binding. Trends in Cognitive Science, 20(7), 482-483. doi:http://dx.doi.org/10.1016/j.tics.2016.04.005

Sui, J., He, X., \& Humphreys, G. W. (2012). Perceptual effects of social salience: evidence from self-prioritization effects on perceptual matching. Journal of Experimental Psychology. Human Perception and Performance, 38(5), 1105-1117. doi:10.1037/a0029792

Sui, J., \& Humphreys, G. W. (2015a). The Integrative Self: How Self-Reference Integrates Perception and Memory. Trends in Cognitive Science, 19(12), 719-728. doi:10.1016/j.tics.2015.08.015

Sui, J., \& Humphreys, G. W. (2015b). The interaction between self-bias and reward: Evidence for common and distinct processes. Q J Exp Psychol (Hove), 68(10), 1952-1964. doi:10.1080/17470218.2015.1023207 
Sui, J., \& Humphreys, G. W. (2015c). More of me! Distinguishing self and reward bias using redundancy gains. Atten Percept Psychophys, 77(8), 2549-2561. doi:10.3758/s13414-0150970-x

Sui, J., Ohrling, E., \& Humphreys, G. W. (2016). Negative mood disrupts self- and reward-biases in perceptual matching. Quaterly Journal of Experimental Psychology (Hove), 69(7), 14381448. doi:10.1080/17470218.2015.1122069

Sui, J., Yankouskaya, A., \& Humphreys, G. W. (2015). Super-capacity me! Super-capacity and violations of race independence for self- but not for reward-associated stimuli. Journal of Experimenta Psychology. Human Perception and Performance, 41(2), 441-452. doi:10.1037/a0038288

Townsend, J. T., \& Eidels, A. (2011). Workload capacity spaces: a unified methodology for response time measures of efficiency as workload is varied. Psychon Bul \& Rev, 18(4), 659681. doi:10.3758/s13423-011-0106-9

Townsend, J. T., \& Wenger, M. J. (2004). A theory of interactive parallel processing: new capacity measures and predictions for a response time inequality series. Psychological Review, 111(4), 1003-1035. doi:10.1037/0033-295X.111.4.1003

Turk, D. J., Brady-van den Bos, M., Collard, P., Gillespie-Smith, K., Conway, M. A., \& Cunningham, S. J. (2013). Divided attention selectively impairs memory for self-relevant information. Memory and Cognition, 41(4), 503-510. doi:10.3758/s13421-012-0279-0

Voss, A., Nagler, M., \& Lerche, V. (2013). Diffusion models in experimental psychology: a practical introduction. Experimental Psychology, 60(6), 385-402. doi:10.1027/16183169/a000218

Voss, A., Rothermund, K., \& Voss, J. (2004). Interpreting the parameters of the diffusion model: an empirical validation. Memory and Cognition, 32(7), 1206-1220.

Voss, A., \& Voss, J. (2007). Fast-dm: a free program for efficient diffusion model analysis. Behavior Research Methods, 39(4), 767-775. 
Voss, A., Voss, J., \& Lerche, V. (2015). Assessing cognitive processes with diffusion model analyses: a tutorial based on fast-dm-30. Frontiers in Psychology, 6, 336. doi:10.3389/fpsyg.2015.00336

Whisman, M. A., Sheldon, C. T., \& Goering, P. (2000). Psychiatric disorders and dissatisfaction with social relationships: does type of relationship matter? Journal of Abnormal Psychology, 109(4), 803-808.

Wildegger, T., Riddoch, J., \& Humphreys, G. W. (2015). Stored color-form knowledge modulates perceptual sensitivity in search. Attention, Perception and Psychophysics, 77(4), 1223-1238. doi:10.3758/s13414-015-0853-1

Wimmer, G. E., \& Buchel, C. (2016). Reactivation of Reward-Related Patterns from Single Past Episodes Supports Memory-Based Decision Making. Journal of Neuroscience, 36(10), 2868-2880. doi:10.1523/JNEUROSCI.3433-15.2016

Witt, J. K., Taylor, J. E., Sugovic, M., \& Wixted, J. T. (2015). Signal Detection Measures Cannot Distinguish Perceptual Biases from Response Biases. Perception, 44(3), 289-300. doi:10.1068/p7908

Yankouskaya, A., Sui, J., Moradi, Z., Rotshtein, P., \& Humphreys, G. W. (2017). Applications of capacity analysis into social cognition domain. In N. Altieri, Fific, M., Little, D., Yang, C. (Ed.), System Factorial Technology. Academic Press.

Yankouskaya, A., Palmer, D., Sui, J, \& Humphreys, G. (2016). Self-bias modulates saccadic control. Quarterly Journal of Experimental Psychology, 68(4), 1-25. http://dx.doi.org/10.1080/17470218.2016.1247897

Zhan, Y., Chen, J., Xiao, X., Li, J., Yang, Z., Fan, W., \& Zhong, Y. (2016). Reward Promotes SelfFace Processing: An Event-Related Potential Study. Frontiers in Psychology, 7, 735. doi:10.3389/fpsyg.2016.00735 